\title{
The control of near-wall turbulence through surface texturing
}

\author{
R García-Mayoral, G Gómez-de-Segura and C T Fairhall \\ Department of Engineering, University of Cambridge, Cambridge CB2 1PZ, UK \\ E-mail: r.gmayoral@eng.cam.ac.uk
}

\begin{abstract}
Certain surfaces that exhibit small textured features can interact with near-wall turbulence and reduce drag, which is of great interest to industries in the aerospace, naval, transport and energy sectors. This paper reviews and discusses the dynamic mechanisms at play in that interaction. General principles of application across different technologies are discussed, and the parameters of interest and relevance are identified. It is argued that the main effect of these surfaces can be expressed as an offset between the positions of the virtual, equivalent smooth walls perceived by different parts of the flow, namely by the mean velocity profile and by the background turbulence, which remains otherwise smooth-like. Individual technologies are also reviewed, including superhydrophobic surfaces, riblets and permeable substrates, with particular emphasis on physical mechanisms that are specific to each technology. We discuss the capillary waves that form in superhydrophobic surfaces and the spanwiseelongated rollers that form over riblets and permeable surfaces.
\end{abstract}

\section{Introduction}

This paper focuses on the effect on wall-bounded turbulence of surfaces that are intentionally textured to manipulate the flow, typically to modify the drag compared to a smooth surface. By texture we understand surface features with a typical lengthscale, $L$, which is either uniform or varies along the surface over distances much larger than itself. Such surfaces are generally well suited for homogenization techniques (Zampogna and Bottaro; 2016; Lācis and Bagheri; 2017), and their effect on the overlying flow is collective, in the sense that the background turbulence does not perceive the detail of each individual texture element, but some form of averaged effect.

We restrict ourselves to the effect of these surfaces on canonical flows, such as flows in channels and pipes and boundary layers with zero or mild pressure gradients, and focus on how they alter the otherwise canonical near-wall turbulence over smooth surfaces. In the discussion we will mostly use results from direct numerical simulations (DNSs) in channels. These are simpler than boundary layers in that the flow does not evolve in the streamwise direction, so for instance the friction Reynolds number and the viscous scales are homogeneous along the domain. Results in channels are of application 
to boundary layers provided that the streamwise evolution is slow enough that the flow is quasi-homogeneous at any given streamwise coordinate, which requires that the mean pressure gradient be small. We will use $x, y$, and $z$ for the streamwise, wall-normal, and spanwise coordinates, and $u, v$, and $w$ for the corresponding velocity components. We will refer to the the channel half-height, the pipe radius or the boundary layer height as the flow thickness, $\delta$, and use a ' + ' superscript to indicate scaling in viscous units, i.e. with the kinematic viscosity $\nu$ and the friction velocity $u_{\tau}=\sqrt{\tau_{w}}$, derived from the tangential stress at the wall $\tau_{w}$.

The first part of the paper is dedicated to the effect of textures in general, when the texture size is small. In section 2 we discuss the effect of the texture in the flow far from the wall, which can be reduced to a shift in the mean velocity profile, independently of how the texture produces that effect. How the surface produces that shift, and how it should be measured, are discussed in section 3. The two sections aim to provide some guidelines that can help unify the interpretation of results by expressing them in the simplest form possible. As the texture becomes larger, the flow starts perceiving its granular nature. We will discuss this effect in the context of superhydrophobic surfaces, which are reviewed in section 4 , although this effect can be expected to be general across other types of surfaces.

The second part of the paper reviews some specific surfaces, namely the aforementioned superhydrophobic surfaces of section 4, riblets in section 5 and permeable surfaces in section 6 . These sections will focus mainly on the dynamics of flow features which are particular to each surface, and that appear in addition to the homogenized effect of the texture, as the surface selects and excites certain modes in the flow dynamics. The review of such mechanisms is non-exhaustive, but intends to illustrate the increased complexity of the flow when textures become large, with dynamical effects that go beyond the mere modulation of near-wall turbulence. In section 4 we will review the effect of capillary waves in the stability of entrapped gas pockets in superhydrophobic surfaces. In section 5 we will cover the onset of KelvinHelmholtz rollers just above a riblet surface when the riblet grooves are sufficiently large, which causes a saturation and eventual degradation of performance. Anisotropic permeable surfaces, a novel, promising technology that could potentially supersede riblets for aircraft applications, are presented in section 6. The paper concludes with some final remarks in section 7 .

\section{The effect of small surface manipulations on turbulence and drag}

Let us assume for simplicity that the surface features can be characterized by a single lengthscale $L$. When $L$ is small, the direct effect on the flow is confined to the nearwall region, as it decays exponentially with the wall-normal height, $y$, as $\sim \mathrm{e}^{-y / L}$ (Seo et al.; 2015). According to the classical theory of wall turbulence, the only effect farther from the wall is a constant shift $\Delta U^{+}$in the velocity profile, while both the Kármán constant $\kappa \approx 0.4$, and the 'wake' function are unaffected (Clauser; 1956). While changes 
in drag are Reynolds-number dependent, $\Delta U^{+}$has extensively been discussed as being universal, so long as the surface parameters remain the same in viscous units (Luchini; 1996; Spalart and McLean; 2011; García-Mayoral and Jiménez; 2011a), i.e. $L^{+}$constant in our case. In the logarithmic region and above we then have

$$
U^{+}=\frac{1}{\kappa} \log y^{+}+B+\Delta U^{+},
$$

where $B$ includes both the near-wall intercept for a canonical, smooth-wall flow, and the contribution from the wake component. The dependence of $\Delta U^{+}$on $L^{+}$at large Reynolds numbers is affected by the modulation of the local viscous lengthscale by the intensity of the outer scales in the flow (Mathis et al.; 2009; Zhang and Chernyshenko; 2016), although this effect is typically second order and will be neglected here. The shift $\Delta U^{+}$produced by some active surfaces has also been shown to be essentially independent of the Reynolds number and to fully characterize the surface effect (Gatti and Quadrio; 2016). It is also widely used in roughness literature, where it is often called the 'roughness function' or 'Hama constant' (Jiménez; 2004; MacDonald et al.; 2017) and has reversed sign, as the shift of the velocity profile is generally to lower values, resulting in a drag increase. Equation (1) can be used to estimate the change in drag caused by a given $\Delta U^{+}$. In external flows, the friction coefficient $c_{f}$ is typically based on the free stream velocity, $U_{\delta}$,

$$
c_{f, \mathrm{BL}}=2 \tau_{w} / U_{\delta}^{2}=2 / U_{\delta}^{+^{2}}
$$

while in internal flows it is more frequent to define it in terms of the flow rate, that is, of the bulk velocity, $U_{B}$,

$$
c_{f, \mathrm{CH}}=2 \tau_{w} / U_{\delta}^{2}=2 / U_{B}^{+2} .
$$

The drag reduction, $D R$, is typically defined as the relative decrease in $c_{f}$ compared to that for a smooth wall, $c_{f, 0}$,

$$
D R=-\frac{\Delta c_{f}}{c_{f, 0}}
$$

The question has often arisen of what corresponding position to choose for the reference smooth wall. Luchini (1995) showed that this choice is essentially irrelevant for external flows, for which the whole boundary layer is simply shifted vertically when the origin is changed. In internal flows, in contrast, the choice can be crucial, as it implies a change in the hydraulic radius, and $c_{f}$ has a very strong dependence on it -through a fifth power in turbulent pipes, for instance. Taking the example of riblets, they can be conceived as ridges protruding from the reference wall or as grooves carved into it. If the riblet height is not several orders of magnitude smaller than $\delta$, the difference in drag due to the difference in hydraulic radius in the reference case could be comparable to the actual effect of the riblets, or even larger. If an experiment or simulation is conducted matching the value of $\delta^{+}$for the target application, then the reference case should obviously be dictated by the application. If for instance, the aim is to add a coating to the internal side of an already-existing pipe, the actual hydraulic radii of the original and final configurations should be taken into account. 


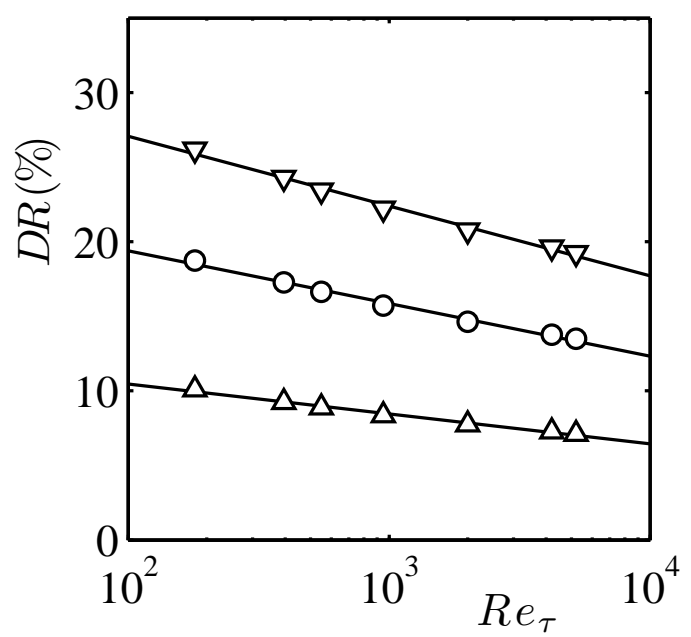

Figure 1. Degradation of $D R$ with the Reynolds number for constant $\Delta U^{+}$, according to (5). The reference velocity is the centerline velocity from the numerical channels of Moser et al. (1999), Hoyas and Jiménez (2006), Lozano-Durán and Jiménez (2014), and Lee and Moser (2015). $\backsim-\Delta U^{+}=1 ; \multimap-, \Delta U^{+}=2 ;-\nabla-, \Delta U^{+}=3$.

On the other hand, experiments and simulations are often conducted at a reduced scale, with $L^{+}$matching the envisaged applications, but $\delta^{+}$substantially smaller, and often only one or two orders of magnitude greater than $L^{+}$. Furthermore, simulations are frequently done in channels for their simplicity, but with a view to boundary-layer applications. Under these circumstances, direct measurements of $D R$ can be misleading. This is in part due to the measurements being very sensitive to differences of order $\sim L$ in the hydraulic radius, while the differences would be negligible in the case of application, but also to the dependence of $D R$ on the Reynolds number through $U_{\delta}^{+}$or $U_{B}^{+}$. This would leave $\Delta U^{+}$as the only relevant measure in scaled-down settings. Measuring $\Delta U^{+}$requires comparing the velocity profiles of the smooth and the textured surface sufficiently away from the wall, at least well into the logarithmic region, which leaves the outstanding question of where to set the height origin for the texture. This will be discussed in section 3.

Even if $D R$ is not a convenient measure for the performance of a surface across different flow configurations and conditions, it is ultimately the figure of industrial and application relevance. It can be obtained from (4) and either (2) or (3). Using the subscript zero to indicate smooth-wall flows, in boundary layers we would have $U_{\delta}^{+}=U_{\delta, 0}^{+}+\Delta U^{+}$. For internal flows, and at the large Reynolds numbers of applications, the contribution to the bulk velocity from the very-near-wall region, where (1) might not hold, is negligible, so we would also have $U_{B}^{+}=U_{B, 0}^{+}+\Delta U^{+}$. The drag reduction is then given by

$$
D R=1-\left(\frac{1}{1+\Delta U^{+} / U_{\delta, 0}^{+}}\right)^{2},
$$

where $U_{\delta, 0}^{+}$would be substituted by $U_{B, 0}^{+}$for internal flows if $c_{f}$ based on the bulk 
velocity was used. Linearized versions of (5) where proposed in Luchini (1996) and García-Mayoral and Jiménez (2011a), but these can only be expected to be accurate for $D R \lesssim 10 \%$, and are probably not needed given the simplicity of (5). Estimates for the change in $D R$ with the friction Reynolds number, $\operatorname{Re}_{\tau}=\delta^{+}$, are given in figure 1 up to Reynolds numbers of industrial relevance, $\operatorname{Re}_{\tau} \approx 10,000$. The figure shows that, although the decrease in $D R$ is logarithmic, it can be of order $\sim 1 / 3$ between measurements at low $\operatorname{Re}_{\tau}$ and applications. On the positive side, further decreases can be expected to be smaller if the logarithmic trend is extrapolated up to $\operatorname{Re}_{\tau} \approx 100,000$.

On a final note on the ambiguity of $D R$ measurements at low $\operatorname{Re}_{\tau}$, significant differences can be observed depending on the setup of both experiments and simulations. For instance, in numerical periodic channels with texture, the drag force on the surfaces cannot be defined unambiguously unless the cross-sectional area is uniform along $x$ (MacDonald et al.; 2018). In addition, channel simulations are often run at constant flow rate or constant pressure gradient, letting the other fluctuate over time about its statistically steady value. Frohnapfel et al. (2012) have discussed in depth the significance of these different setups. For certain applications, the flow rate needs to meet a constant demand, so the first option would be most suited. If, instead, the pressure loss along the duct is capped by the pumping technology, the second option would be preferable. Frohnapfel et al. (2012) also considered a third option, constant power input, which would be suitable when the power available to impel the fluid is the limiting factor, as is often the case in hydraulic applications. However, the distinction between these different setups is once more relevant when the simulations match the conditions of the target application, particularly the Reynolds number. Extrapolating the results to higher Reynolds numbers can again be misleading. For instance, simulations at constant $U_{B}$ with drag reduction can experience significant variations in $\operatorname{Re}_{\tau}=\delta u_{\tau} / \nu$, as the variation in $c_{f}$ in (3) can only arise from variations in $\tau_{w}=u_{\tau}^{2}$. If the reference, smooth-wall flow is at sufficiently low $\mathrm{Re}_{\tau}$, this can result in a relaminarization of the flow. The profound changes in the flow dynamics would then not correlate with the subtler modulation that could be expected at large $\operatorname{Re}_{\tau}$. On the other hand, assuming that a given texture at size $L^{+}$always results in the same $\Delta U^{+}$, results can be still extrapolated to higher Reynolds numbers, and whether $U_{B}$ or $u_{\tau}$ are to remain constant can be considered separately. It should however be noted that the effect of the texture can be expected to depend on the actual value of $L^{+}$, that is, both $L^{+}$and $\Delta U^{+}$would need to be scaled with the friction velocity of the resulting flow, and not that of the reference smooth wall, to remain $\mathrm{Re}_{\tau}$-independent.

\section{Protrusion heights, slip lengths and virtual origins}

The concept of protrusion height was proposed by Bechert and Bartenwerfer (1989) for riblets. Bechert and Bartenwerfer suggested that, taking as reference the plane of the riblet tips, $y=0$, the mean streamwise flow experienced a different virtual origin, at a depth below the tips that they termed protrusion height, $\ell_{u}$. If $L$ is small, notionally 
$L^{+} \ll 1$, the velocity profile in the immediate vicinity of the wall is essentially linear, as in the viscous sublayer over a smooth wall. The concept of protrusion height can then be expressed through an apparent Navier slip condition for the overlying flow,

$$
u=\ell_{u} \partial u / \partial y \text {. }
$$

Elsewhere, for instance in the superhydrophobic-surface community, $\ell_{u}$ is called slip length. We will also refer to the height $y=-\ell_{u}$ as the virtual origin for the streamwise velocity. In wall units, the Navier slip condition for the mean flow is $U^{+}\left(y^{+}=0\right)=\ell_{u}^{+}$, so the concept of slip length $\ell_{u}^{+}$is often used interchangeably with that of slip velocity, $U_{s}^{+}=U^{+}\left(y^{+}=0\right)$.

Luchini et al. (1991) noticed that the characterization of the surface through $\ell_{u}$ was incomplete, and proposed that a spanwise protrusion height, $\ell_{w}$, was also required. The underlying idea is that a texture that changes drag does so by causing different apparent origins for the mean flow and for the turbulent fluctuations and eddies, particularly for the quasi-streamwise vortices of the near-wall cycle. If the vortices experience a virtual origin above that experienced by the mean flow, they are effectively pushed away from the 'wall' or, more specifically, with respect to the plane were the mean $U(y)$ goes to zero, $y=-\ell_{u}$. As the vortices are displaced away from the wall, the near-wall turbulent mixing of streamwise momentum is reduced. Since this mixing is responsible for the high local wall shear (Orlandi and Jiménez; 1994), the result is a lower skin friction. Luchini et al. (1991) proposed that the virtual origin perceived by the vortices can be defined through a spanwise protrusion height so that, if the vortices perceive the origin at $y=-\ell_{w}$, the corresponding boundary condition at $y=0$ is $w=\ell_{w} \partial w / \partial y$. From the mean momentum equation for $U(y)$, it can be inferred that the shape of the velocity profile is determined by the dynamics of wall turbulence through the Reynolds shear stress. Let us assume that the only effect of the texture on the turbulence dynamics is setting their origin, that is, the 'wall' that they perceive, at $y^{+}=-\ell_{w}^{+}$. It follows that the only change in the mean velocity profile, compared to a smooth-wall, will be a shift by its value at $y^{+}=-\ell_{w}^{+}$, that is, $\Delta U^{+}=\ell_{u}^{+}-\ell_{w}^{+}$, which will propagate to any height $y^{+}$above. Note that, for this to hold, the granular, direct effect of the texture on the mean flow and the turbulence dynamics needs to be negligible, so only the homogenized effects, i.e. the slip lengths $\ell_{u}^{+}$and $\ell_{w}^{+}$, are significant. This implies that, in principle, the requirement $L^{+} \ll 1$ can be relaxed to $L^{+}$being small enough that turbulent eddies, and the mean flow, only perceive its homogenized effect. This will be further discussed in section 4. Luchini et al. (1991) proposed that the relevant parameter for riblet drag reduction was $\ell_{u}-\ell_{w}$. Based on arguments consistent with the discussion in this paragraph, Jiménez (1994) and Luchini (1996) proposed that $\Delta U^{+} \propto \ell_{u}^{+}-\ell_{w}^{+}$, with a constant of proportionality between 0.6 and 1 depending on the author (see also Bechert et al. (1997a) and Luchini (2015)).

However, the adverse effect of $\ell_{w}^{+}$on $\Delta U^{+}$saturates for values of $\ell_{w}^{+}$of just a few wall units. In the context of superhydrophobic surfaces, and following Min and Kim (2004), Busse and Sandham (2012) conducted an extensive series of simulations of channels with 

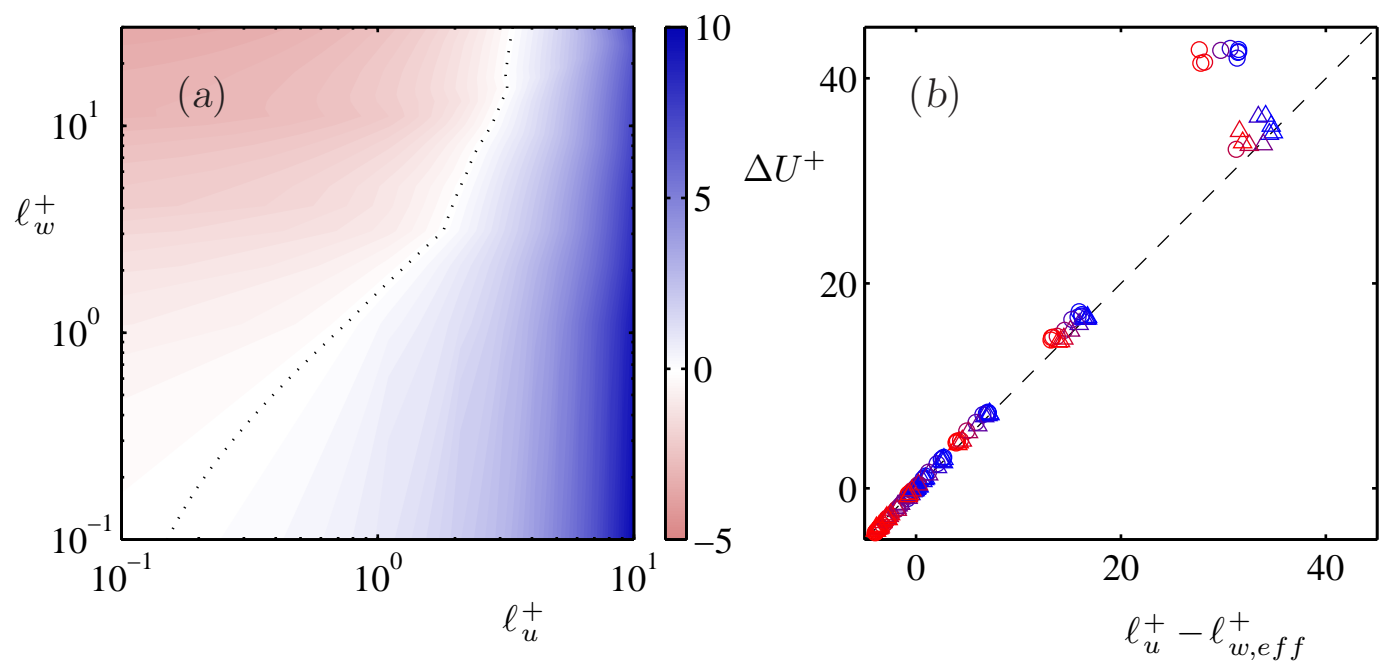

Figure 2. Results for $\Delta U^{+}$from the simulations with homogeneous $\ell_{u}^{+}$and $\ell_{w}^{+}$from Busse and Sandham (2012). (a) Contours of $\Delta U^{+}$in the $\left(\ell_{u}^{+}, \ell_{w}^{+}\right)$parametric space for $\operatorname{Re}_{\tau} \approx 360 ; \cdots \cdots \cdot \cdot$, neutral drag curve, $\Delta U^{+}=0$. (b) Collapse of the same data vs. $\ell_{u}^{+}-\ell_{w, \text { eff }}^{+}$; $\mathrm{O}$, simulations at $\operatorname{Re}_{\tau} \approx 180, \triangle, \mathrm{Re}_{\tau} \approx 360$; colors indicate, blue to red, $\ell_{w}^{+} \approx 10^{(-2,-1: 0.5: 2,3)} ;----, \Delta U^{+}=\ell_{u}^{+}-\ell_{w, \text { eff }}^{+}$. Note that variables have been scaled with the viscous units of the corresponding simulations. Figure adapted from Fairhall and García-Mayoral (2018).

$\ell_{u}^{+}$and $\ell_{w}^{+}$ranging from $\sim 0.01$ to $\sim 100$ wall units. Results from their simulations are compiled in figure 2. Busse and Sandham (2012) proposed empirical formulas, based on Fukagata et al. (2006), that accounted for the non-symmetry of the drag reduction with respect to $\ell_{u}^{+}$and $\ell_{w}^{+}$shown in figure 2(a). In the framework of Luchini's theory of protrusion heights, the results of Busse and Sandham (2012) can be more simply interpreted by introducing an empirical, effective spanwise slip length, $\ell_{w, e f f}^{+}$(Fairhall and García-Mayoral; 2018),

$$
\ell_{w, e f f}^{+}=\frac{\ell_{w}^{+}}{1+\ell_{w}^{+} / 4}
$$

to account for the saturation of the effect of $\ell_{w}^{+}$. Figure $2(b)$ shows the excellent collapse of $\Delta U^{+}$from the simulations of Busse and Sandham (2012) with the law

$$
\Delta U^{+}=\ell_{u}^{+}-\ell_{w, e f f}^{+} .
$$

The only notable deviation is for simulations at $\operatorname{Re}_{\tau} \approx 180$ with $\ell_{u}^{+} \sim 100$, which is in this case $\sim \delta^{+}$. This deviation can probably be explained by surfaces with such large $\ell_{u}^{+}$ perturbing the whole flow thickness, so the results are not extrapolable to the general case of higher Reynolds numbers, for which most of the flow would remain unperturbed. The behavior at $\operatorname{Re}_{\tau} \approx 360$, accordingly, agrees well with (8).

The cause for the saturation of the effect of $\ell_{w}^{+}$has been recently investigated by Gómez-de-Segura et al. (2018a). The assumption underlying the protrusion height theory is that quasi-streamwise vortices induce a Couette-like, transverse shear over the virtual origin that they perceive, but no wall-normal velocity. This relies on the property 
that $w$ is linear just above the wall while, from continuity, $v$ is quadratic. To first order, therefore, a displacement to $y=-\ell_{w}$ of the virtual origin that vortices perceive could be represented at the boundary plane, $y=0$, through a Robin condition for the spanwise velocity, $w=\ell_{w} \partial w / \partial y$, plus impermeability for the wall-normal velocity, $v=0$. In Gómez-de-Segura et al. (2018a), it was argued that the displacement of the vortices, on average, towards $y^{+}=0$ would necessarily saturate eventually, unless the shift of the origin perceived by $w, \ell_{w}^{+}$, was accompanied by a corresponding shift for $v, \ell_{v}^{+}$. In general, when the virtual origins perceived by $v$ and $w$ differ, the quasi-streamwise vortices would experience an intermediate virtual origin, $\ell_{T}^{+}$. If impermeability for $v$ holds at $y^{+}=0$, the virtual origin for turbulence is the observed $\ell_{T}^{+}=\ell_{w, \text { eff }}^{+}$for slip-only simulations. Preliminary research was conducted on methods to impose a virtual origin for $v$ as a homogeneous boundary condition, the most successful being the implementation of a Robin boundary condition. These would have the same structure as slip conditions for $u$ and $w$, and match the ratio between $v$ and $\partial v / \partial y$ at a height $\ell_{v}$ above a smooth wall. When this method was used to model a virtual origin for $v$ coincident with that for $w$, $\ell_{v}^{+}=\ell_{w}^{+}$, no saturation of the spanwise slip effect was observed, and $\ell_{T}^{+}=\ell_{w}^{+}$.

Mean velocity profiles and other flow statistics are compiled in figures 3 and 4 for simulations with different virtual origins for $u, v$ and $w$ from Fairhall and GarcíaMayoral (2018) and Gómez-de-Segura et al. (2018a). The figures show the collapse obtained when the height $y^{+}=-\ell_{T}^{+}$is used as the origin, with all viscous magnitudes using the corresponding $u_{\tau}$. This $u_{\tau}$ is obtained by extrapolating the linear curve of total stress to $y^{+}=-\ell_{T}^{+}$, although in the range of $\ell_{T}^{+}$considered, and at $\operatorname{Re}_{\tau} \approx 180$, the effect of the shift on $u_{\tau}$ is small. For comparison, the figures also include results portrayed in the conventional form, taking $y^{+}=0$ as the origin and scaled with $u_{\tau}$ measured at that height. The results are both from simulations with non-zero $\ell_{u}^{+}$and $\ell_{w}^{+}$and zero $v$ at $y=0$, so that $\ell_{T}^{+} \approx \ell_{w, e f f}^{+}$, and from a simulation with non-zero $\ell_{u}^{+}$, $\ell_{w}^{+}$and $\ell_{v}^{+}$, all roughly equal to $\ell_{T}^{+} \approx 4$. The different behavior in the latter case is qualitatively noticeable in the panels with origin at $y^{+}=0$, and can be attributed to the quasi-streamwise vortices penetrating closer to the boundary plane, as the matching of $\ell_{v}^{+}$with $\ell_{w}^{+}$impedes the saturation observed in slip-only simulations. However when the results take as reference $y^{+}=-\ell_{T}^{+}$, all the simulations collapse. The question of how to determine $\ell_{T}^{+}$in general remains open, but our preliminary work suggests that, at least for $\ell_{T}^{+} \lesssim 5$, it could be found a posteriori by matching the curves for $v^{\prime+}, w^{\prime+}$, the Reynolds stress $\tau_{u v}^{+}$, and the streamwise vorticity $\omega_{x}^{\prime+}$, as well as the mean velocity profile, in the active region of the near-wall cycle, $y^{+}-\ell_{T}^{+} \approx 10-25$, to those of smoothwall turbulence. For simulations with $v=0$ at $y=0$ this results in $\ell_{T}^{+} \approx \ell_{w, e f f}^{+}$, and for simulations with $\ell_{v}^{+} \approx \ell_{w}^{+}$it results in $\ell_{T}^{+} \approx \ell_{w}^{+}$. Nevertheless, further work is needed to find a more general form.

The collapse observed in figures 3 and 4 supports the idea that the near wall cycle, and the turbulence dynamics above, remain essentially canonical, that is, maintain the same dynamics as over a smooth wall, and that the effect of the virtual origins on turbulence can be accounted for through the shift $\ell_{T}^{+}$alone. This includes both 

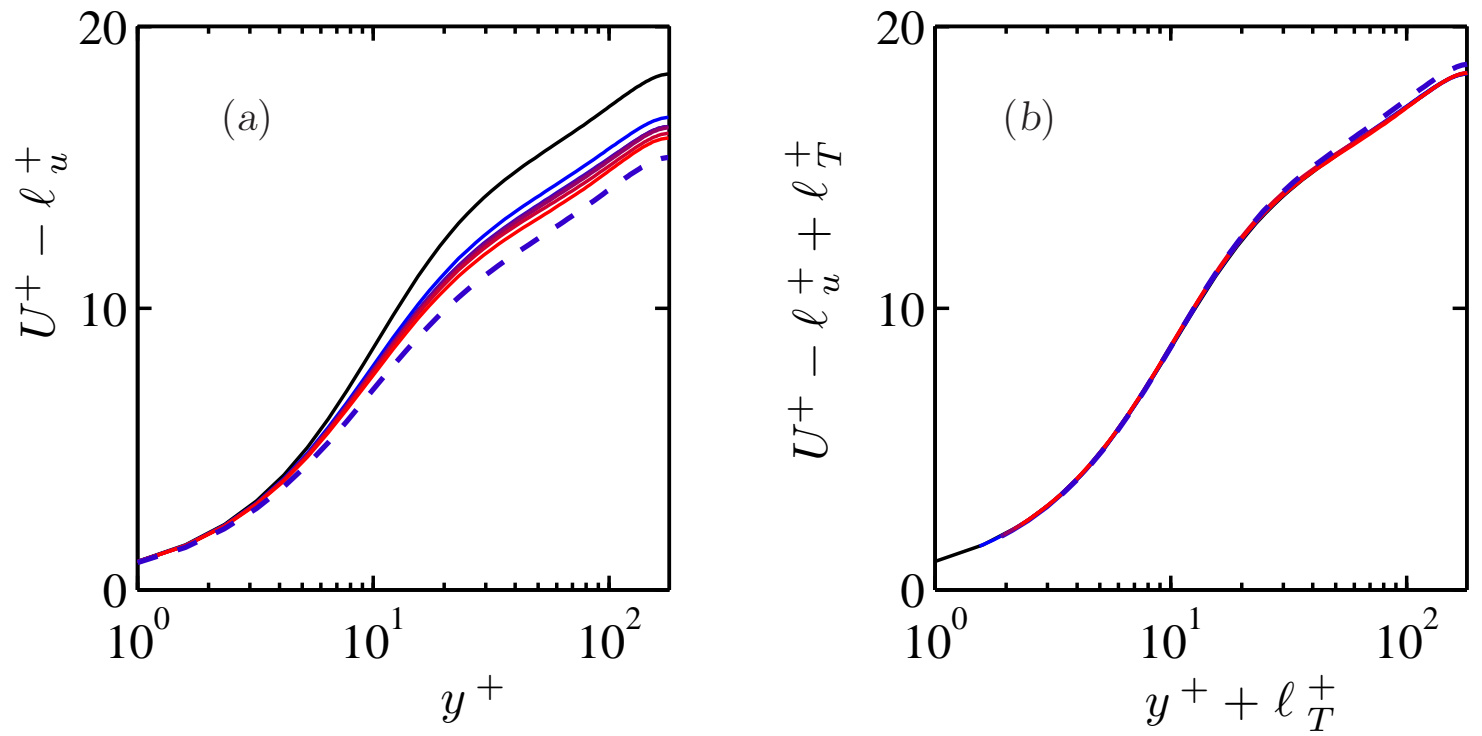

Figure 3. Mean velocity profiles for simulations with slip and transpiration to model different virtual origins for $u, v$ and $w$, from Fairhall and García-Mayoral (2018) and Gómez-de-Segura et al. (2018a). — simulations with zero transpiration and, blue to red, $\left(\ell_{u}^{+}, \ell_{w}^{+}\right) \approx(4,3),(4,4),(6,4),(7,4),(9,5)$, and $(10,6)$, with the black line corresponding to the smooth-wall case; ----, simulation with $\ell_{u}^{+}, \ell_{w}^{+}$and $\ell_{v}^{+}$ approximately equal to $\ell_{T}^{+}=4$. (a) Profiles portrayed with the origin at the plane where boundary conditions are imposed, $y^{+}=0$, and scaled with $u_{\tau}$ measured at that plane. (b) Profiles portrayed shifted to the virtual origin for turbulence $\ell_{T}^{+}$, and scaled with the corresponding $u_{\tau}$.
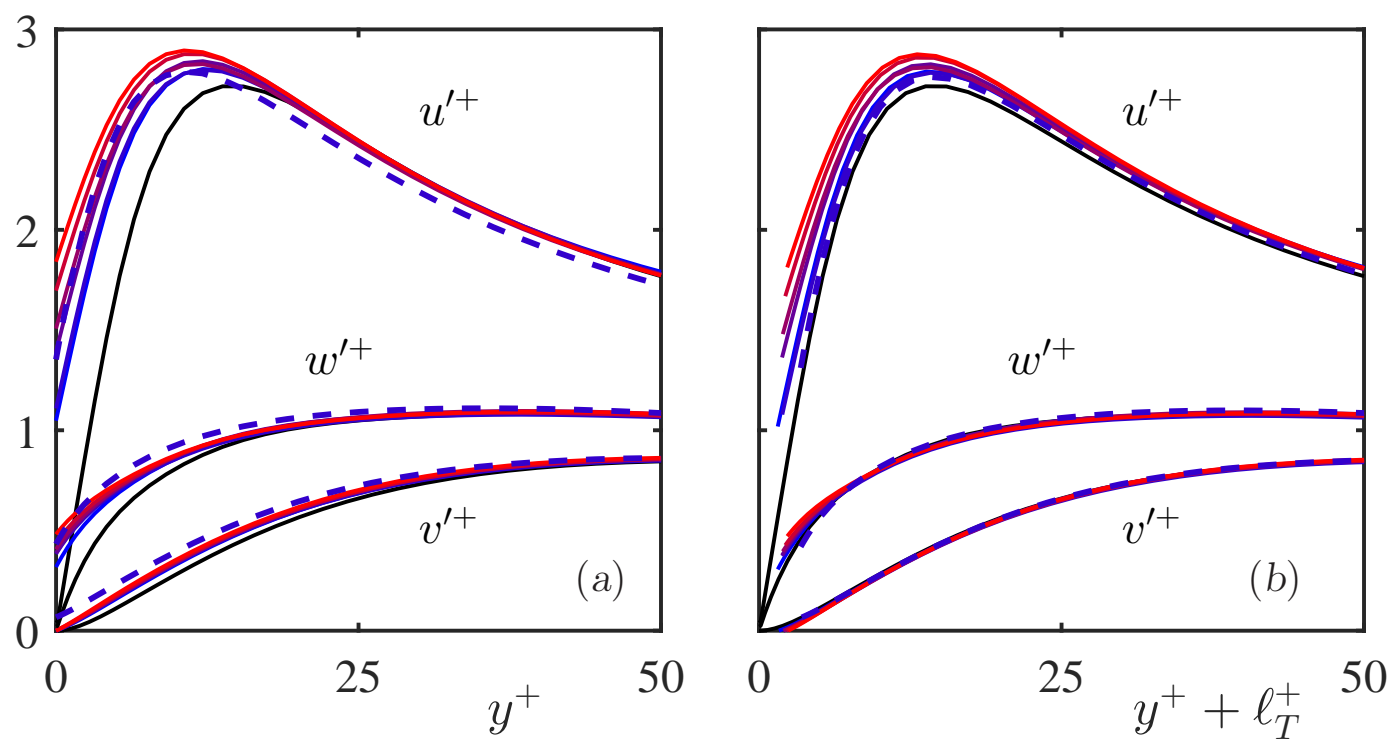

Figure 4. Rms velocity fluctuations for the simulations of figure 3, represented with the same line styles. (a), with the height origin at the plane where boundary conditions are imposed, $y^{+}=0$, and scaled with $u_{\tau}$ measured at that plane; $(b)$, with the height origin shifted by $\ell_{T}^{+}$, and scaled with the corresponding $u_{\tau}$. 
the changes in magnitude and the shifts towards the plane $y=0$ that have been conventionally reported in the literature (Min and Kim; 2004; Busse and Sandham; 2012) and which can also be observed in figure 4(a).

Figure 3 compares the mean velocity profiles for the different simulations of Fairhall and García-Mayoral (2018) and Gómez-de-Segura et al. (2018a) with that for a smooth wall. We should note that setting different origins for the profile can result in different apparent slopes in the logarithmic region, which can be interpreted as a variation in $\kappa$ (see for instance Mizuno and Jiménez (2011) and references therein). The difference can be appreciated in the expansion, for $y^{+}>\ell_{T}^{+}$, of $\log \left(y^{+}+\ell_{T}^{+}\right)$as $\log \left(y^{+}\right)+\ell_{T}^{+} / y^{+}$. The correction $\ell_{T}^{+} / y^{+}$eventually vanishes for large $y^{+}$, but can be significant for a substantial portion of the logarithmic layer, and manifest as an apparent change in $\kappa$ if $\operatorname{Re}_{\tau}$ is not sufficiently large. The figure suggests that setting the origin using $\ell_{T}^{+}$recovers the correct slope in the logarithmic region, although the logarithmic layer barely exists at theses low Reynolds numbers and further simulations would be needed to confirm this. Figure $3(b)$ indicates that, with the proposed scaling, the only difference for $U^{+}$between the different cases would be the shift $\ell_{u}^{+}-\ell_{T}^{+}$. This provides the answer to the question raised in section 2 of where to set the height origin for the textured surface. From the virtual origin $y^{+}-\ell_{T}^{+}=0$, and with the corresponding scaling, the velocity profile is smooth-like, particularly far enough from the surface and into the log region, except for the shift

$$
\Delta U^{+}=\ell_{u}^{+}-\ell_{T}^{+} .
$$

The plane $y^{+}-\ell_{T}^{+}=0$ should therefore be the virtual origin used to define the position of a reference smooth wall and the friction velocity.

As we have discussed, $\ell_{T}^{+}$plays a fundamental roll in the displacement of the quasistreamwise vortices relative to the plane $y=0$, and is essentially determined by $\ell_{v}$ and $\ell_{w}$. In turn, $\ell_{u}$ plays an important roll in determining the shift of the mean flow, but is relatively inactive with respect to the turbulent cycle. These differences appear in the flow rms fluctuations portrayed in figure 4(b). The curves for $v^{\prime+}, w^{\prime+}$ collapse with the smooth-wall data at all heights. In contrast, $u^{\prime+}$ collapses well above $y^{+}-\ell_{T}^{+} \gtrsim 15$, but deviates below this height, as the profiles of $u^{\prime+}$ decay linearly towards their respective origins, at $y^{+}=-\ell_{u}^{+}$. The only case that collapses with the smooth one in this region is, therefore, the one that has $\ell_{u}^{+}=\ell_{T}^{+}$. For $\ell_{u}^{+}>\ell_{T}^{+}$, there is an increased $y^{+}$-range where viscosity acts to bring $u^{\prime+}$ quasi-linearly to zero, and the local gradient is therefore smaller. This is likely responsible for the slight increase of the peak value near $y^{+}-\ell_{T}^{+} \gtrsim 15$ observed in figure $4(b)$

In summary, textured surfaces where the texture is small enough to be perceived as a homogeneous surface by the flow, can be modeled as presenting different virtual origins for the different velocity components, $\ell_{u}^{+}, \ell_{v}^{+}$, and $\ell_{w}^{+}$. The virtual origin perceived by the quasi-streamwise vortices, $\ell_{T}^{+}$, is set by the latter two, and sets the scale for the overlying turbulence, which otherwise remains smooth-like. Luchini's theory of protrusion heights can then be generalized to the shift $\Delta U^{+}$produced being given by the difference between 
$\ell_{u}^{+}$and $\ell_{T}^{+}$, as expressed in (9).

For a given surface, the texture lengthscale $L^{+}$changes with the viscous lengthscale of the overlying flow, $\nu / u_{\tau}$. As $L^{+}$increases, so do $\ell_{u}^{+}, \ell_{T}^{+}$, and their difference. Therefore, drag-reducing surfaces improve their performance for increasing $L^{+}$. However, the improvement eventually breaks down. One reason is that the flow begins to perceive the texture granularity, so its effect is not merely the homogenized one. This will be discussed in section 4.1 in the context of superhydrophobic surfaces. In addition, larger textures can select and excite additional dynamic modes in the flow. These effects vary greatly depending on the type of surface texture. Examples for superhydrophobic and riblet surfaces are given in sections 4 and 5 .

\section{Superhydrophobic surfaces}

Superhydrophobicity enables textured surfaces immersed in water to entrap pockets (or bubbles) of air. The bubbles can lodge within the texture grooves, when the groove size is small enough. This is known as the Cassie-Baxter state. In the opposite, fully wetted condition, or Wenzel state (Wenzel; 1936), the surface cavities are filled with water, and the hydrophobic effect is lost. Under Cassie-Baxter conditions, much of the overlying water flow is in contact with the entrapped air, instead of with the solid surface. The air layer acts then as a lubricant for the outer flow, which can effectively slip over the wall, experiencing reduced friction compared to conventional, smooth surfaces (Rothstein; 2010). Ou and Rothstein (2005) showed experimentally that, under turbulent conditions, superhydrophobic surfaces are able to achieve high drag reductions, of up to at least 25\%. Min and Kim (2004) studied the reduction for turbulent flows numerically, modeling the superhydrophobicity of the surface as a homogeneous slip length, which implies that the surface texture modeled is isotropic. To account for texture anisotropy, Busse and Sandham (2012) conducted DNSs with different streamwise and spanwise slip lengths, with the results discussed in 3 . The detailed geometry of the texture was first studied in simulations by Martell et al. (2009), who used a patterned slip/no-slip boundary condition to model the alternating contact with the entrapped air pockets and the roughness crests.

Although the drag-reducing properties of superhydrophobic surfaces on turbulent flows have received a great deal of attention recently, both experimentally ( $\mathrm{Ou}$ and Rothstein; 2005; Gogte et al.; 2005; Daniello et al.; 2009; Park et al.; 2014; Ling et al.; 2016; Gose et al.; 2018) and numerically (Min and Kim; 2004; Martell et al.; 2009, 2010; Park et al.; 2013; Türk et al.; 2014; Jelly et al.; 2014), the interaction of these surfaces with the flow is not yet fully understood. Most experimental measurements reported have been conducted at texture sizes of order $L^{+} \approx 0.5-5$. Although no clear reason is often given, it is likely that for larger textures the stability of the bubbles is lost, and with it the drag-reducing effect. In contrast, numerical simulations have often been conducted at $L^{+} \sim 100$, and only more recently have simulations with $L^{+} \sim 10$ been conducted. This is due to a compromise between computational cost and physical 
fidelity, but it is not clear if some of the dynamics that are dominant in the former range of $L^{+}$are also important at the smaller sizes of real applications. It is also questionable whether the air bubbles would remain stable at such large $L^{+}$, were their stability not forcefully imposed through the numerical model. In particular, the above cited simulations assumed that the gas-liquid interfaces maintained a perfectly flat, rigid shape. The reduction predicted in numerical studies increases monotonically with $L^{+}$, in agreement with the theoretical predictions of Lauga and Stone (2003), Fukagata et al. (2006), and Ybert et al. (2007), although the behavior deviates from theory for large textures. In real flows, however, the superhydrophobic effect would be completely lost for sufficiently large grooves, once the Cassie-Baxter state is lost and the surface fully wetted (Aljallis et al.; 2013). Progress is currently being made on the fabrication of hierarchical (Lee and Kim; 2011b,a) and gas-pocket-restoring (Lee and Kim; 2012) surfaces to minimize the impact dewetting effects.

Some work in the literature focuses on possible degrading effects, which would eventually lead to the depletion of the gas pockets, but the mechanisms that cause the degradation remain largely unknown. Hyväluoma and Harting (2008) and Teo and Khoo (2010) studied the effect of bubble shape when the interface is not perfectly flat, while Busse and Sandham (2013) have analyzed how the performance degrades as the air layer is lost, and the texture crests begin to protrude out of it, introducing a roughness-like effect. Lee and Kim (2009), Patankar (2010), and Samaha et al. (2011) have analyzed the transition from the Cassie-Baxter to the Wenzel state through static pressure effects, while Aljallis et al. (2013) and Liu et al. (2016) have considered the effect of shear on bubble depletion, the latter in the context of liquid-infused surfaces.

Here, we will use superhydrophobic surfaces to illustrate the effect of texture granularity on the slip properties of a surface, and discuss the additional dynamic effects caused by the interface deformability at larger $L^{+}$.

\subsection{The effect of texture on slip}

Some of the studies on the effect of $\ell_{u}^{+}$and $\ell_{w}^{+}$discussed in section 3 where conducted in the framework of superhydrophobic surfaces. It is unfortunate that the connection with Luchini's protrusion height theory and the concept of an offset between virtual origins for $u$ and $w$ (Jiménez; 1994; Luchini; 1996) was not properly established, given that both mechanisms are actually the same (Luchini; 2015).

Homogeneous slip conditions of the form of equation (6) were used to model and study the superhydrophobic effect in the simulations of Min and Kim (2004), Fukagata et al. (2006), and Busse and Sandham (2012). They have also been used to study dynamic effects within the gas layer (Busse et al.; 2013; Aghdam and Ricco; 2016). However, these models neglect the irregularity in the surface wetted by the overlying water flow, which is made up of the gas-liquid interface interspersed by the solid protrusions of the rough surface, which act as pylons onto which the interface attaches.

To model the alternating contact with the entrapped air pockets and the roughness 


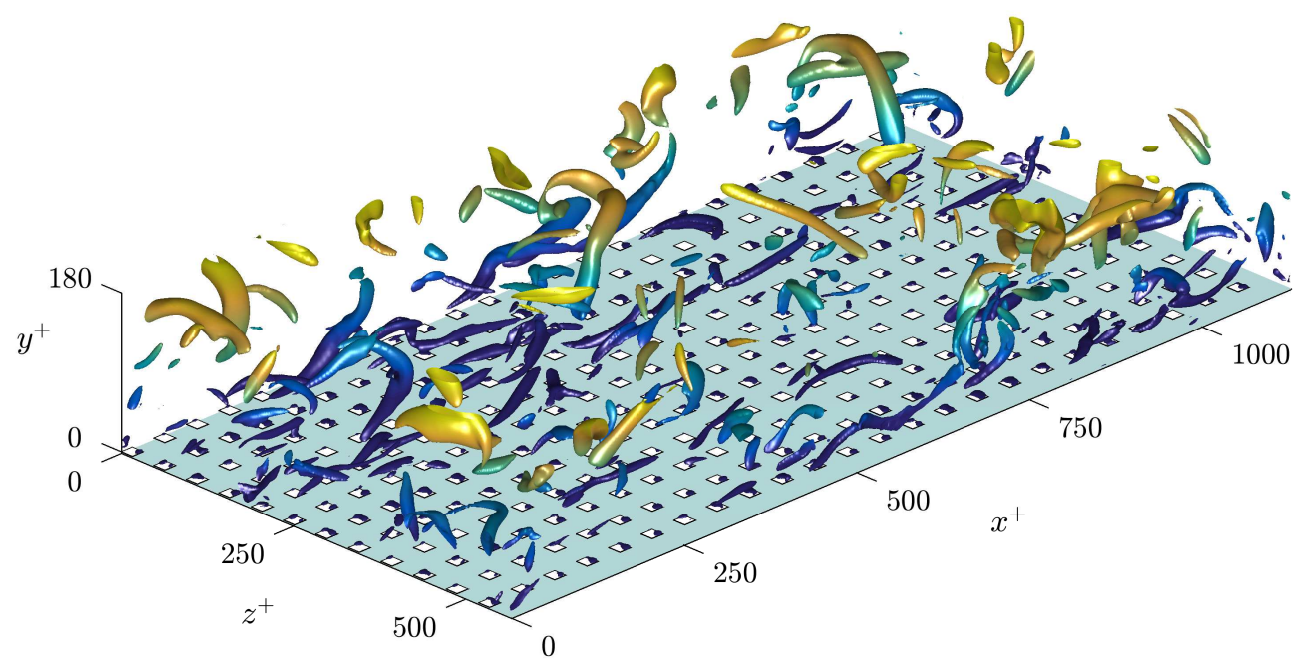

Figure 5. Instantaneous realization of vortical structures, using the $\mathrm{Q}$ criterion, in a direct simulation of turbulence over a modeled textured superhydrophobic surface with $L^{+} \approx 47$ from Fairhall and García-Mayoral (2018). The surface is modeled as a no slip boundary at the solid post crests, in white, and a free slip surface at the air -water interface, in light blue.

crests, Martell et al. (2009) introduced a patterned free-slip/no-slip boundary condition. Figure 5 illustrates the flow over an instance of such modeled surface. This approach does not account for the finite viscosity of the gas and the corresponding finite shear at the liquid/gas interface (Schönecker et al.; 2014), nor for the surface not being perfectly flat, but subject to deformation either statically (Hyväluoma and Harting; 2008; Teo and Khoo; 2010; Busse and Sandham; 2013; Seo et al.; 2015) or dynamically (Seo et al.; 2018). Nevertheless, it provides a first-order approximation to the granular effect of the texture, and it has become widely adopted (Park et al.; 2013; Jelly et al.; 2014; Türk et al.; 2014; Seo et al.; 2015; Rastegari and Akhavan; 2015; Seo and Mani; 2016; Fairhall and García-Mayoral; 2018).

Seo and Mani (2016), Fairhall and García-Mayoral (2018) and Fairhall and GarcíaMayoral (2017) used the above model to study the effect of the surface granularity, by comparing simulations with square posts in an equispaced arrangement, as shown in figure 5, with simulations with equivalent uniform slip lengths, $\ell_{x}^{+}$and $\ell_{z}^{+}$. Seo and Mani (2016) reported that the homogenized slip length model broke down for texture sizes of order $L^{+} \gtrsim 10$, which yielded $\ell_{x}^{+} \approx \ell_{z}^{+} \approx 4$ in the configurations studied. The breakdown was caused by a loss of correlation between the tangential velocities and the corresponding shears, when averaged over a texture-periodic unit, for which a perfect correlation would have the form of (6). The traditional, qualitative argument is that this breakdown occurs when $L^{+}$becomes comparable to the lengthscales in the overlying turbulence, which therefore no longer perceive the surface as homogeneous. To 

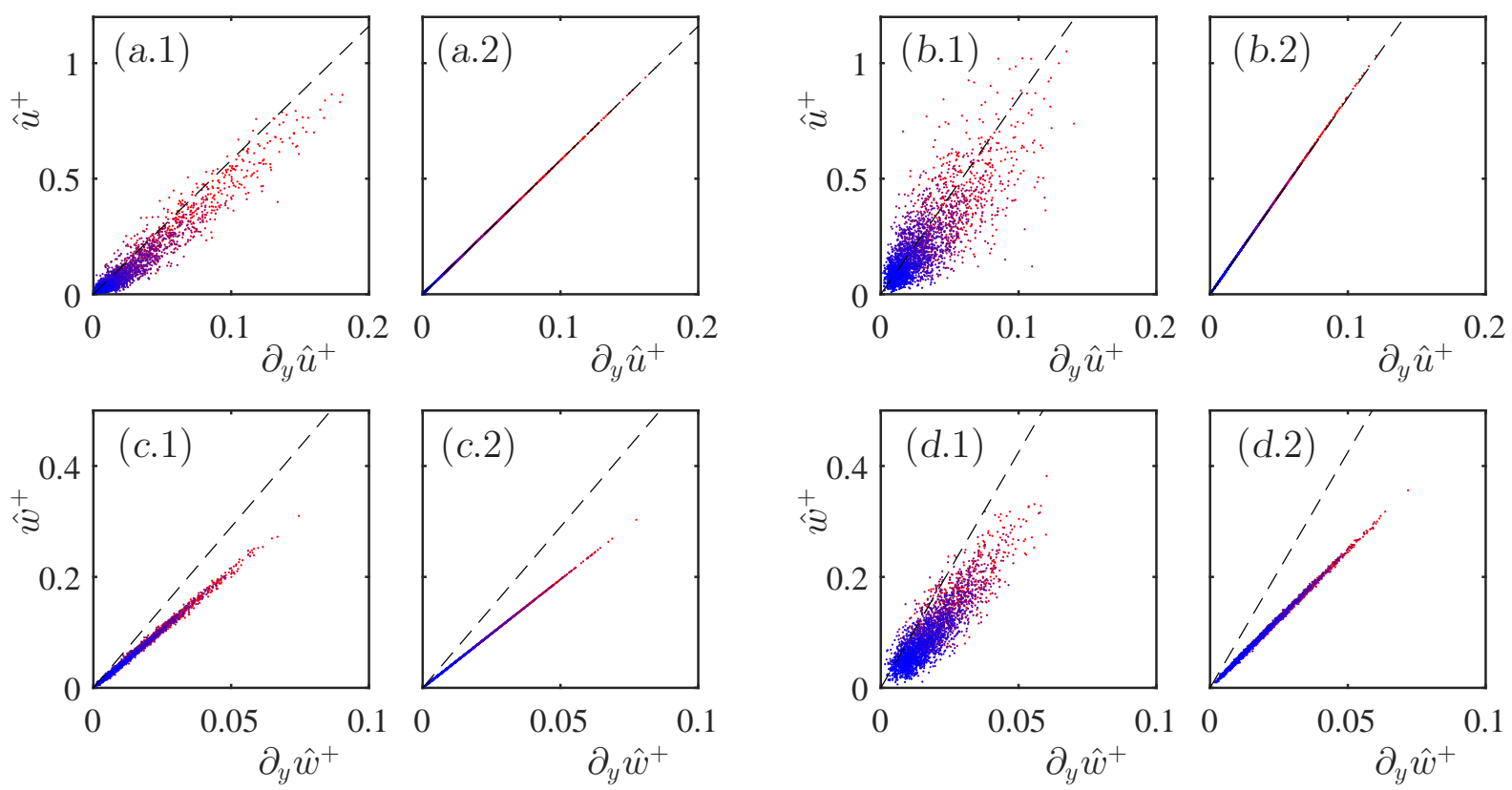

Figure 6. Instantaneous correlation between the magnitudes of the streamwise and spanwise velocity and shear at the interface plane $y=0$ for modeled textured superhydrophobic surfaces from Fairhall and García-Mayoral (2018). (a) and (c), $L^{+} \approx 18$; $(b)$ and $(d), L^{+} \approx 35$. Panels labeled ' 1 ' portray results from the full flow signal, and panels labeled ' 2 ' results for the background turbulence component alone. Results are for Fourier modes with wavelengths $\lambda_{z}^{+}=94$ and 113 and, blue to red, $\lambda_{x}^{+}=103-1131$. The dashed line corresponds to the value of $\ell_{x}^{+}$observed for the mean flow $U(y)$.

investigate the validity of this assertion, Fairhall and García-Mayoral (2018) analyzed the correlation in Fourier space, as shown in figure 6. They could then discriminate different flow lengthscales, and test whether, at large $L^{+}$, flow lengthscales much larger than $L^{+}$still experienced a uniform slip condition. Their results showed that this was not the case, as the velocity-shear correlation was lost across all lengthscales. Fairhall and García-Mayoral (2018) suggested that this was caused by the singularity introduced by the step change from the exposed surface of solid posts, with no slip, to the fluid interface, with free shear. The Fourier transform of a step change is dense, so the signature of the texture scrambles into the whole Fourier space, and is not restricted to wavelengths of order $\sim L^{+}$.

However, it has been shown that, for small textures, the flow can be decomposed into a background turbulent signal, essentially free from a texture signature, and a texture-coherent component, with the latter modulated in amplitude by the former (Abderrahaman-Elena and Garcia-Mayoral; 2016). This is similar to conventional triple decomposition (Reynolds and Hussain; 1972), except for the amplitude modulation, which is necessary to minimize the footprint of the texture on the background signal. Fairhall and García-Mayoral (2018) argued that the texture-coherent component was responsible for the loss of shear/velocity correlation, and in Fairhall and García-Mayoral 

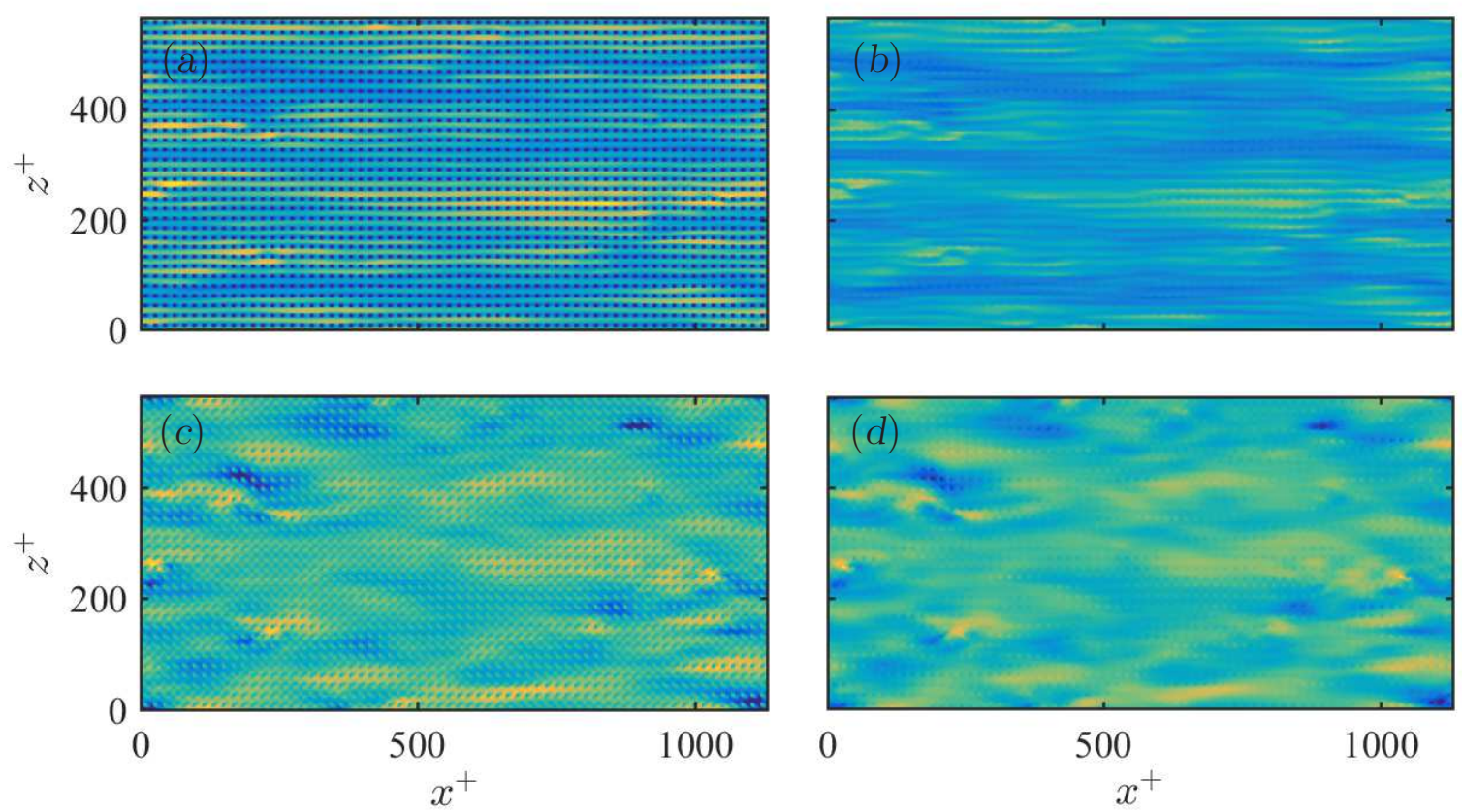

Figure 7. Instantaneous realizations of velocity at the interface plane $y=0$ for a modeled textured superhydrophobic surface with $L^{+} \approx 18$, from Fairhall and GarcíaMayoral (2018). (a), full $u$ signal; (b) background turbulent signal for $u$, removing the contribution from the texture-coherent flow, induced and modulated in amplitude by the background signal. (c), full $w$ signal; $(d)$ background turbulent signal for $w$, as in (b)

(2017) they showed that this component carried all the uncorrelated part of the velocities and shears. The extraction of the background component from the full signal is illustrated in figure 7 for $L^{+} \approx 18$. For smaller $L^{+}$, the result is similar to spectral filtering, but for this texture size the lengthscales of the texture and the smallest features of the overlying turbulence overlap, so spectral filtering would also remove part of the background signal. Fairhall and García-Mayoral (2017) showed that, when the texturecoherent component is removed, the correlation between velocities and shears in the background turbulent signal is recovered. The correlation is nearly perfect, even for textures as large as $L^{+} \approx 35$, as shown in figure 6 , compared with the lack of correlation in the full flow signal. Therefore, slip lengths could unambiguously be defined for the background, turbulent flow.

Fairhall and García-Mayoral (2017) subsequently studied whether such slip lengths were sufficient to model the effect of the superhydrophobic surface on turbulence. To compare with texture-resolving simulations, they conducted a set of simulations with homogeneous slip conditions, selected so they matched $\ell_{u}^{+}$and $\ell_{w}^{+}$obtained from the textured-resolving ones. These homogeneous-boundary simulations have been discussed 

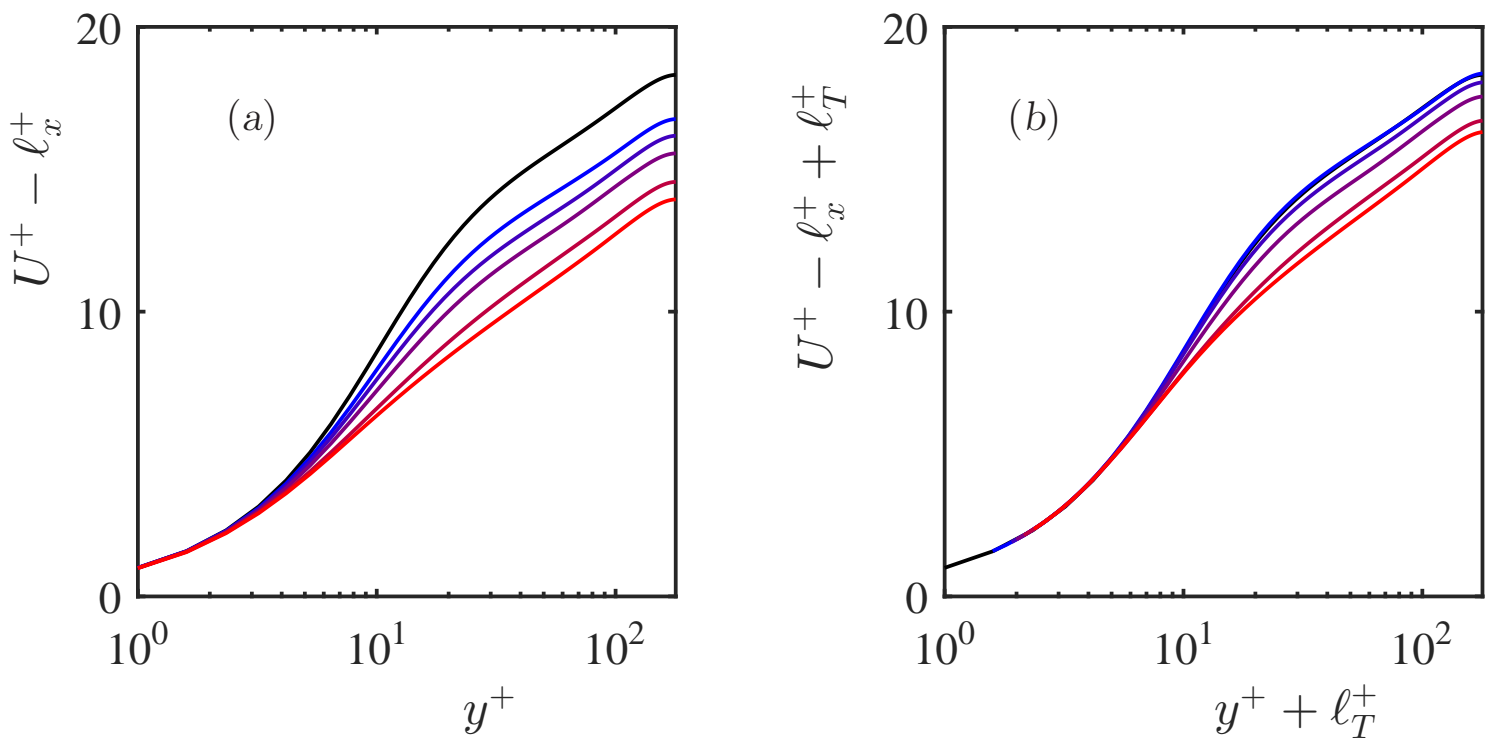

Figure 8. Mean velocity profiles for simulations of textured superhydrophobic surfaces from Fairhall and García-Mayoral (2018). Blue to red, $L^{+} \approx 12,18,24,35$, and 47, with the black line corresponding to the smooth-wall case. (a) Profiles portrayed with the origin at the plane where boundary conditions are imposed, $y^{+}=0$, and scaled with $u_{\tau}$ measured at that plane. (b) Profiles portrayed shifted to the virtual origin for turbulence $\ell_{T}^{+}$, and scaled with the corresponding $u_{\tau}$.
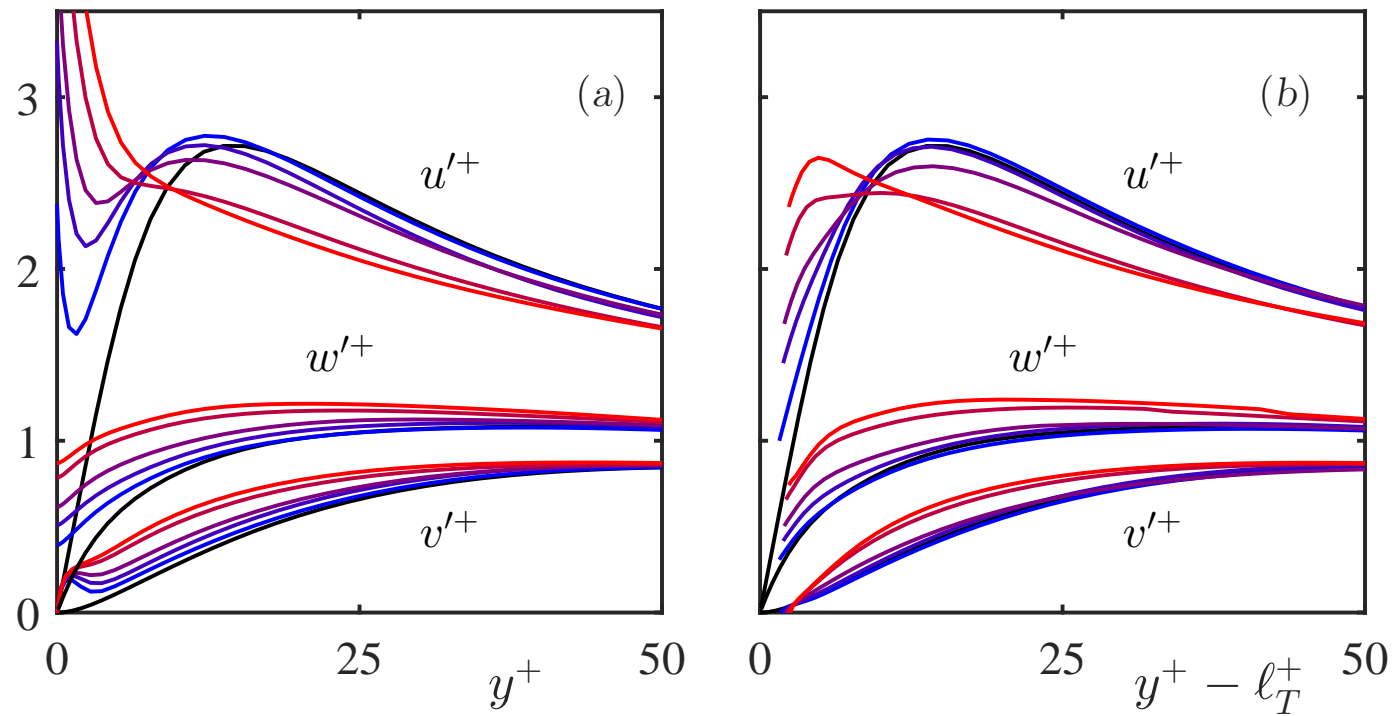

Figure 9. Rms velocity fluctuations for the simulations of figure 8 , represented with the same line styles. (a), with the height origin at the plane where boundary conditions are imposed, $y^{+}=0$, and scaled with $u_{\tau}$ measured at that plane; $(b)$, with the height origin shifted by $\ell_{T}^{+}$, and scaled with the corresponding $u_{\tau}$. 
in section 3, where it was shown that turbulence remained canonical, smooth-wall-like, save for the offset of apparent origin to $y^{+}=-\ell_{T}^{+}$. Results for the corresponding textureresolving simulations are shown in figures 8 and 9 . Figure 8 portrays mean velocity profiles that can be compared directly with those of figure 3. The downward trend with increasing $L^{+}$of the profiles with origin at $y^{+}=0$ is qualitatively similar to that observed for homogeneous slip, but more pronounced. The results with origin at $y^{+}=-\ell_{T}^{+}$, in turn, show deviations from the smooth-wall profile that increase with $L^{+}$. These deviations can also be appreciated in the rms fluctuations portrayed in figure 9. Once the fluctuations are rescaled to have the origin at $y^{+}=-\ell_{T}^{+}$, simulations with $L^{+} \lesssim 20$ show good agreement with the smooth-wall data. For $L^{+} \approx 24$, a small deviation can be observed, but for $L^{+} \gtrsim 30$ the deviations become more profound and the fluctuations can no longer be ascribed to those of canonical near-wall turbulence. These results suggests that, for $L^{+} \lesssim 25$, the effect the texture on the background turbulence essentially reduces to setting a virtual origin below the actual surface, through a homogenized slip effect that can be expressed through a slip length $\ell_{w}^{+}$. For $L^{+} \gtrsim 30$, however, the effect of the texture can no longer be reduced to an effective slip boundary condition. The flow decomposition of Abderrahaman-Elena and Garcia-Mayoral (2016) suggests that any further effect is caused by the non-linear coupling of the texture-coherent and the background-turbulence components. This coupling can only occur through the advective terms in Navier-Stokes equations, and its signature cannot be extracted when filtering out the terms due exclusively to the texture-coherent flow. The effect of the texture extends then beyond merely imposing a boundary condition, and can be interpreted as a forcing in the fluid equations for the background turbulence in the near-wall region.

\subsection{The effect of interface deformability}

The effect of the texture granularity discussed in section 4.1 is adverse, but is intimately associated to the ability to produce a slip-length, given that, at least in the vanishingly small limit, slip lengths are obtained from solving the flow over the detailed texture (Luchini et al.; 1991; Lauga and Stone; 2003; Ybert et al.; 2007). As the texture size increases, other dynamical mechanisms arise that can degrade drag further. For superhydrophobic surfaces, some of these mechanisms are caused by the deformability of the air/water interfaces. These deformability is governed by the surface tension $\sigma$ through the force balance across the interface, the Young-Laplace equation. Its linearized form is

$$
\nabla^{2} \eta \approx \frac{\Delta p}{\sigma}
$$

where $\eta$ is the interface height and $\Delta p$ the local, instantaneous pressure jump across the interface. The effect of this deformability was studied in Seo et al. (2015) and Seo et al. (2018).

In Seo et al. (2015), the stationary interface deformation caused by the timeaveraged flow was studied. This flow has stagnation regions just before and after the posts, causing a texture-coherent distribution of low and high pressure that can be 


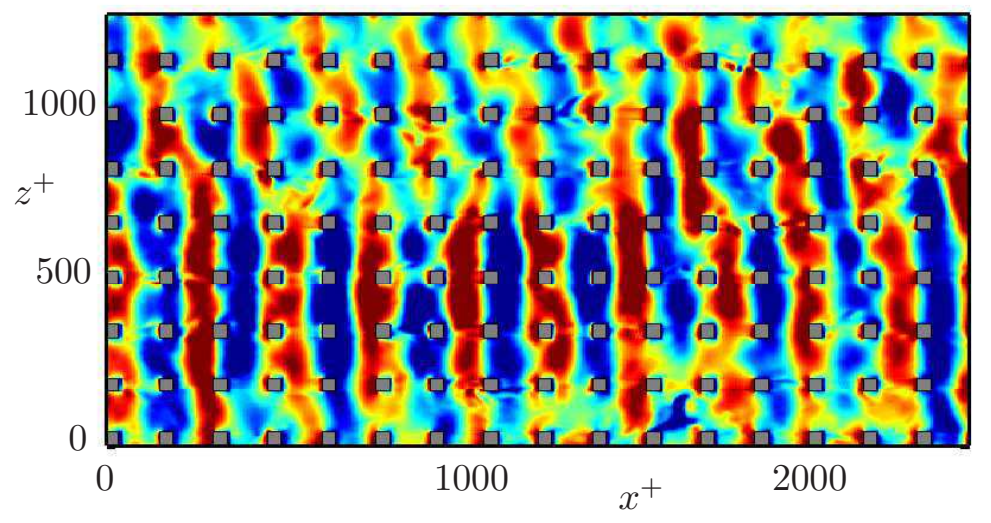

Figure 10. Instantaneous realization of the fluctuating pressure at $y^{+}=0$ for a modeled textured superhydrophobic surface with finite surface tension, from Seo et al. (2018). Blue to red, $p^{+}=-20-20$.

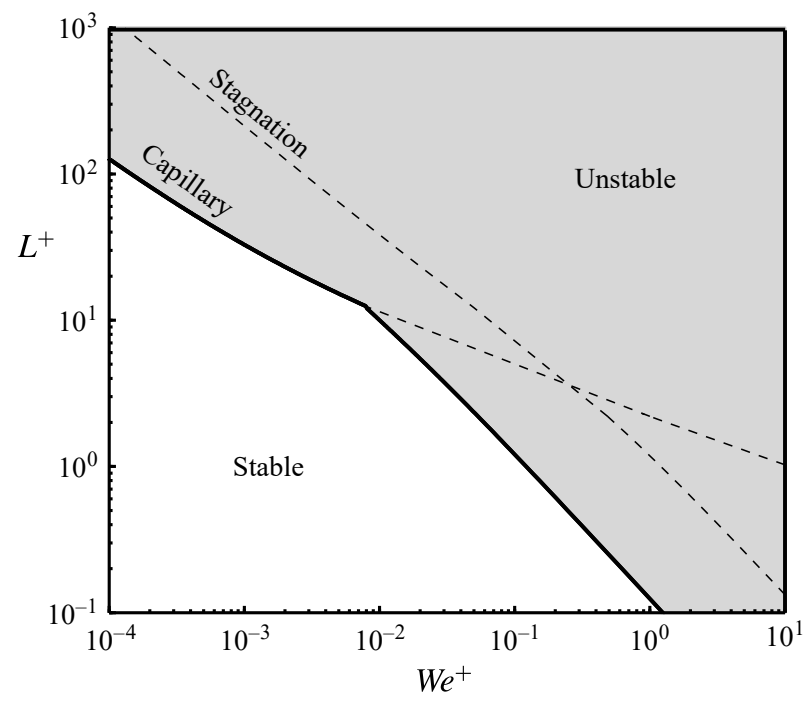

Figure 11. Schematic map for the stability of water/air interfaces in superhydrophobic surfaces as a function of the texture size $L^{+}$and the Weber number $\mathrm{We}^{+}$, adapted from Seo et al. (2018), reproduced with permission. The limit parallel to that for stagnation accounts for the overlapped effect of pressure fluctuations from the background turbulence.

observed in figure 10. Seo et al. (2015) analyzed how the resulting deformation would scale with $L^{+}$, the slip velocity $U_{s}^{+}$and the Webber number $\mathrm{We}^{+}=\rho u_{\tau} \nu / \sigma$, and used the results to estimate when the maximum contact angle admissible by the interface would be exceeded. This provided bounds for realizable texture sizes $L^{+}$at different flow conditions, given by $\mathrm{We}^{+}$, beyond which the entrapped gas pockets could not be expected to remain stable.

In Seo et al. (2018), the time-dependent, dynamic coupling of the flow and the interface was implemented into DNSs. The authors found that a dynamical mechanism, separate from canonical near-wall turbulence, developed when the fully-coupled interface 
deformation was considered. This mechanism consisted of spanwise-coherent, interfacial capillary waves propagating upstream, as those shown in figure 10. These are similar to the natural, membrane-like modes of deformation of a two-fluid interface studied in classical literature (Squire; 1953; Taylor; 1959). Seo et al. (2018) again analyzed how the resulting deformation scaled with $L^{+}, U_{s}^{+}$and $\mathrm{We}^{+}$, and use this information to find the limits for textures realizable under failure due to this capillary waves.

Considering the two mechanisms just discussed, the resulting realizability maps are illustrated in the schematic of figure 11. Most applications are at $\mathrm{We}^{+} \approx 10^{-3}-10^{-2}$ (Seo et al.; 2018). In this range, capillary waves would be the leading mechanism for failure, which could be expected to occur for $L^{+} \approx 10-20$. Experiments frequently report drag reduction for $L^{+} \approx 0.5-5$ (Daniello et al.; 2009; Woolford et al.; 2009; Park et al.; 2014), but further and more detailed data is required to determine for what values of $L^{+}$beyond these, and through which mechanisms, gas pockets lose their stability. The theoretical limits discussed here, supported by numerical evidence, provide trends and guidelines to orient future experimental campaigns.

\section{Riblets}

A popular type of surface manipulation is directional roughness, in the form of small two-dimensional protrusions aligned with the flow, which are known as riblets. They have been one of the few turbulent-drag-reduction techniques successfully demonstrated not only in theory, but also in practice, both in the laboratory and in full aerodynamic configurations. A fairly broad early review was that of Walsh (1990b), and more recent ones are those of Choi (2000), who emphasizes the work of the ERCOFTAC drag reduction group, Bushnell (2003), which is oriented towards drag reduction techniques for aircraft, and García-Mayoral and Jiménez (2011a), focused mainly on the flow physics. Jiménez (2004) viewed drag reduction by riblets as a transitional roughness effect.

Riblets of different geometries have been tested in wind tunnels, demonstrating drag reductions of up to $10 \%$ over flat plates. These are lower than those achieved by superhydrophobic surfaces, but while those are restricted to naval applications, riblets can be used in land and air vehicles. Walsh and Lindemann (1984) tested several shapes, including triangular, notched-peak, sinusoidal, and U-shaped riblets, obtaining maximum drag reductions of $7-8 \%$ for riblet spacings of approximately 15 wall units. Riblet experiments have also been conducted in oil channels, which allow larger riblet dimensions and better control of the geometry, although typically at lower Reynolds numbers and shorter development lengths than wind tunnels. For example, Bechert et al. (1997a) conducted extensive tests on blade-shaped and trapezoidal-groove riblets, and proposed the latter as a compromise between optimum performance and practical fabrication and maintenance. Off-design conditions have also been considered, including of yaw (Hage et al.; 2000; García-Mayoral and Jiménez; 2011a) and tip erosion (Walsh; 1990a; García-Mayoral and Jiménez; 2011a). Riblet performance is not significantly 
affected by yaw angles below $10 \mathrm{deg}-15 \mathrm{deg}$, but drag reduction is negated at $30 \mathrm{deg}-$ $35 \mathrm{deg}$, and a drag increase is observed for higher angles, peaking at $90 \mathrm{deg}$. Tip sharpness is critical for a good performance, halving for tip radii of order $10 \%$ of the peak-to-peak spacing.

Riblets have been used successfully to reduce the overall drag of airfoils (Lee and Jang; 2005) and aircraft (Viswanath; 2002), with optimum riblet spacings of the order of $30-70 \mu \mathrm{m}$. Szodruch (1991) reports on the flight tests of a commercial airplane (Airbus 320) with riblets over $70 \%$ of its surface, and estimates an overall $2 \%$ drag reduction, based on the fuel savings obtained. A summary of those tests, including maintenance and durability issues, can be found in Robert (1992). The discrepancy between the optimum laboratory performance and full configurations is probably to be expected from any method based on the reduction of skin friction. Not all the drag of an aircraft is friction (Roskam; 1987), and much of the latter is distributed over three-dimensional or geometrically complex areas where drag control is difficult to optimize. Traditionally, riblets were implemented as an adhesive plastic film, which presented maintenance difficulties that outweighed the benefits obtained. However, recent manufacturing technologies allow simpler implementation and greater control of the micro-geometry (Kordy; 2015), and are being considered for introduction in the next generation aircraft by the main manufacturers.

There is anecdotal evidence of the successful use of riblets in applications other than aircraft, particularly in sporting events in which cost and maintenance considerations are less important than in commercial aviation. The hull of the USA challengers in the America's Cup 1987 and 2010 sailing competitions were fitted with riblets, which had been banned by the regulations in intervening years. Both challengers won the Cup, although it is impossible to determine whether riblets had any real role. Riblets were also used in the 1984 Olympic rowing events, but they were subsequently forbidden in official racing, together with all other devices that "modify the properties of the boundary layer". Racing swimsuits produced by Speedo, TYR and Arena in the early 2000s also employed riblet patterns on the surface to reduce passive drag in competitive swimming (Krieger; 2004), claiming a drag reduction of up to 4\%. Much of that improvement was however most likely due to the reduction of form drag, whose contribution in human swimming is much larger than that of friction (Marinho et al.; 2009). Speedo's latest model, the Fastskin LZR Racer, seems to have dropped riblets completely and turned its focus solely on reducing form drag (Matthews; 2008).

A recurrent theme of riblet research has been the motivation by biological surfaces, which are often geometrically complex. Bruse et al. (1993) conducted oil channel tests on shark-skin replicas, hairy surfaces based on the ideas of Kramer (1937), and riblets with adjustable geometry, and Bechert et al. (1997b) reviewed the drag reduction properties of biological surfaces and their replicas. More recently, Boomsma and Sotiropoulos (2016) conducted DNSs of shark skin denticles, obtaining a net drag increase. In turn, Itoh et al. (2006) conducted experiments on the flow over seal fur, obtaining drag reductions of up to $12 \%$, with a dependence on mean hair separation similar to that of riblets on 


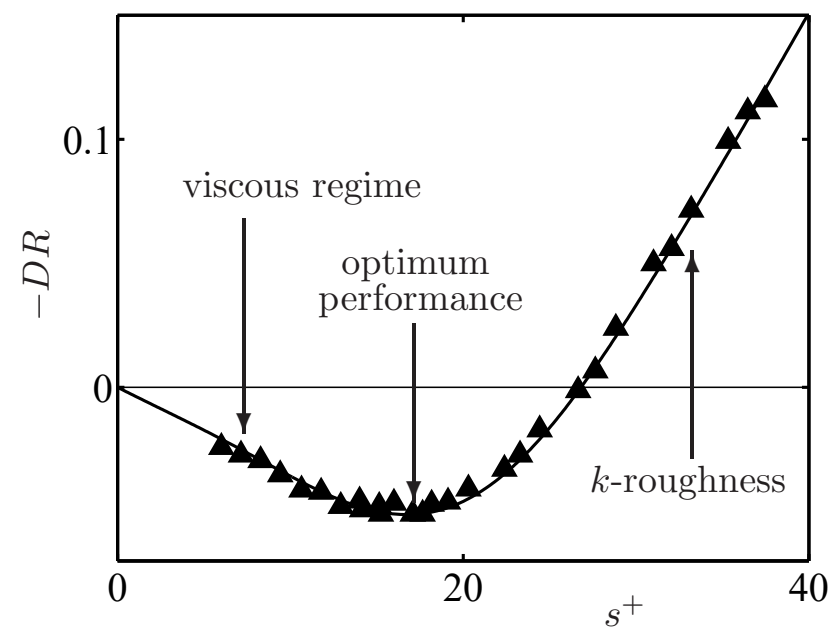

Figure 12. Typical riblet drag reduction curve as a function of the riblet spacing $s^{+}$. Data for a triangular riblet with $60^{\circ}$ peak sharpness, from Bechert et al. (1997a). Figure adapted from García-Mayoral and Jiménez (2011b), reproduced with permission.

peak-to-peak spacing. Instead of the directional roughness of conventional riblets, these hairy surfaces exploit the effect of anisotropic porosity, which will be further discussed in section 6.

Another approach to the design of riblets attempts to combine the drag reduction properties of riblets with those of spanwise oscillation of the wall (Jung et al.; 1992). Viotti et al. (2009) suggested that the oscillating effect could also be produced by imposing at the wall a spanwise velocity profile constant in time, but sinusoidal in the streamwise direction. This kind of velocity distribution could be achieved by implementing three-dimensional riblets, with a slight alternative misalignment with the flow, inducing some coherent streamwise-varying spanwise velocity in the vicinity of the wall. Sha et al. (2005) measured the drag reduction of zigzag riblets, and Kramer et al. (2010) did the same for sinusoidal riblets, but neither achieved results better than those for straight riblets with the same section. The range of oscillation wavelengths and amplitudes tested was however too narrow for that statement to be considered conclusive. More recently, Sasamori et al. (2014) measured an improvement in optimum performance from $\sim 10 \%$ to $\sim 12 \%$ for sinusoidal riblets with an oscillation wavelength of $\sim 360$ wall units.

The physical mechanism of the drag reduction by riblets has been investigated in detail, although some aspects remain controversial. In particular, mean and local velocity profiles and turbulent statistics within and above the riblet grooves have been reported for experiments in wind tunnels (Choi; 1989; Vukoslavcevic et al.; 1992; Park and Wallace; 1994; Lee and Lee; 2001), water channels (Suzuki and Kasagi; 1994), and numerical experiments (Choi et al.; 1993; Chu and Karniadakis; 1993; Goldstein et al.; 1995; Goldstein and Tuan; 1998; El-Samni et al.; 2007; García-Mayoral and Jiménez; 2011b; García-Mayoral and Jiménez; 2012).

Early in the investigation of riblets, Walsh and Lindemann (1984) showed that the 


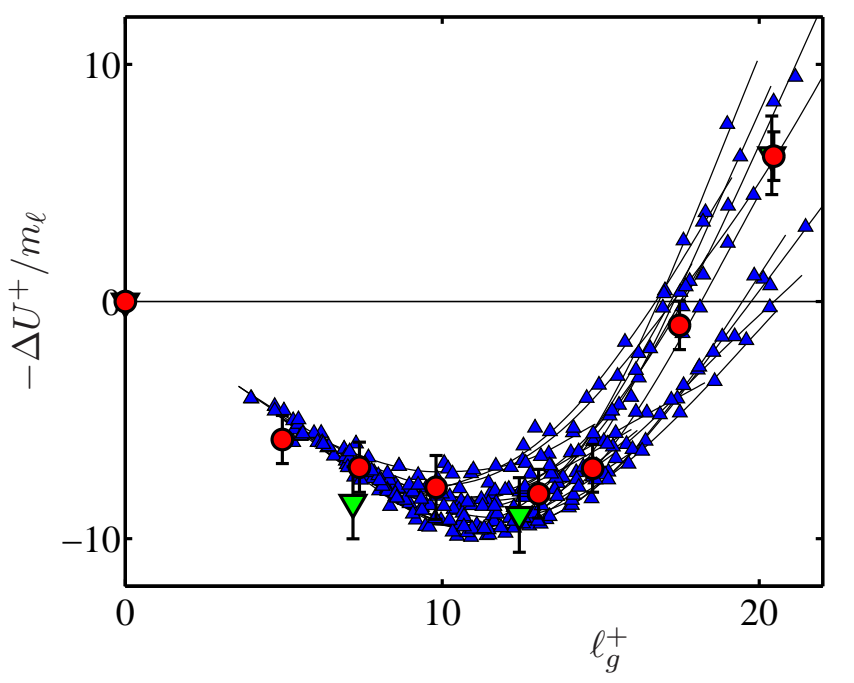

Figure 13. Riblet drag reduction curves as a function of $\ell_{g}^{+}$for several conventional riblets, normalized by their different slopes in the viscous regime. $\triangle$, experimental results for several riblet geometries from Bechert et al. (1997a); O, DNS results from García-Mayoral and Jiménez (2011b); $\nabla$, DNS results from García-Mayoral and Jiménez (2012).

Reynolds number dependence of the effect of a given riblet geometry on the skin friction could be approximately expressed in terms of the riblet dimensions in wall units, $L^{+}$. Historically, a popular measure of the riblet size $L$ has been the groove spacing $s$, but other dimensions, such as the depth $h$, have also been used. The dependence of the performance of a particular riblet geometry on the rib spacing is sketched in figure 12 . The range of small size, where the drag reduction increases linearly, is often termed the 'viscous' regime. As the riblets get larger and their performance saturates, a minimum drag is reached when the viscous regime 'breaks down,' for an optimum spacing $s_{\text {opt }}^{+}$. The viscous limit is fairly well understood and quantified (Luchini et al.; 1991; Luchini; 1996), and the mechanism of its breakdown has been studied in several works (Choi et al.; 1993; Goldstein and Tuan; 1998; García-Mayoral and Jiménez; 2011b; García-Mayoral and Jiménez; 2012). García-Mayoral and Jiménez (2011b) have proposed the square root of the groove cross-section $\ell_{g}^{+}$, as a lengthscale alternative to $s^{+}$to measure riblet size. Using this scaling, the drag curves of diverse conventional riblets are empirically observed to have little variation in their optimal size, $\ell_{g, \text { opt }}^{+} \approx 11$. This collapse can be observed in figure 13. The linear regime and its breakdown are further discussed in the next subsections.

\subsection{The viscous regime}

In the viscous regime of small $s^{+}$, the contribution of the nonlinear terms to the interaction of the flow with the riblets is negligible and the $D R$ depends linearly on $s^{+}$, as mentioned above. The linearity of this regime was recently verified experimentally by Grüneberger and Hage (2011). The regime eventually breaks down for typical spacings 
$s_{\text {opt }}^{+} \approx 10-20$, for which drag reduction is maximum. For even larger riblets, the reduction ultimately becomes a drag increase and follows a typical $k$-roughness behavior (Jiménez; 2004). The optimum performance of each riblet geometry can roughly be estimated as the product of the breakdown size $s_{\mathrm{opt}}^{+}$and the slope of the drag curve in the viscous regime, $m_{s}$ if the riblet size is expressed through $s^{+}$. Both $m_{s}$ and $s_{\mathrm{opt}}^{+}$ depend on the geometry, but the qualitative behavior is always as just described.

García-Mayoral and Jiménez (2011b) reviewed the available experimental evidence and noted that the viscous and breakdown regimes are essentially unrelated phenomena. The drag-reduction mechanism in the viscous regime is governed by the offset of virtual origins that riblets induce for the mean flow and the turbulent fluctuations, as discussed in section 3. There is a thin near-wall region in turbulent flows over smooth walls where viscous effects are dominant, nonlinear inertial effects can be neglected, and the mean velocity profile is linear. Its thickness is $5-10$ wall units (Tennekes and Lumley; 1972). From the point of view of a small protrusion in this layer, the outer flow can be represented as a time-dependent, but otherwise uniform shear. Riblets destroy that uniformity near the wall but, if $s^{+} \ll 1$, the flow still behaves as a uniform shear for $y \gg s$. A further simplification is that the problem decouples into two two-dimensional sub-problems in the $z-y$ cross plane, because the equations of motion are locally linear, the riblets are uniform in the streamwise direction, and the outer shear varies only slowly with $x$ when compared to its variations in the cross plane. The first sub-problem is the longitudinal flow of $u$, driven by a homogeneous streamwise shear, $u \approx S_{x}\left(y+\ell_{u}\right)$ at $y \gg s$. The second sub-problem is the transverse flow of $v$ and $w$, driven by a homogeneous spanwise shear $w \approx S_{z}\left(y+\ell_{w}\right)$ also at $y \gg s$. Far from the wall, the effect of the riblets reduces to the virtual origins $\ell_{u}^{+}$and $\ell_{T}^{+} \approx \ell_{w}^{+}$, through the mechanism that has been discussed in depth in section 3 .

The numerical calculation of the virtual origins only requires the solution of the two stationary two-dimensional Stokes problems for $\ell_{u}$ and $\ell_{w}$, which are computationally much less intensive than the three-dimensional, time-dependent, turbulent flow over ribbed walls. Note that the linearity of the Stokes problems implies that $\ell_{u}, \ell_{w}$ and their difference are all proportional to the riblet size. This formally requires $s^{+} \ll 1$, but in practice holds through the whole viscous regime, $s^{+} \leq 10-15$, as observed in experiments. Equation (9) provides then an expression for the viscous slope $m_{s}$,

$$
\Delta U^{+} \approx \ell_{u}^{+}-\ell_{w}^{+}=\frac{\ell_{u}-\ell_{w}}{s} s^{+}=m_{s} s^{+} .
$$

Conversely, if the size of the riblets is expressed through $\ell_{g}^{+}$, we have

$$
\Delta U^{+} \approx \frac{\ell_{u}-\ell_{w}}{\ell_{g}} \ell_{g}^{+}=m_{\ell} \ell_{g}^{+} .
$$

The latter has the advantage, assuming that the collapse $\ell_{g \text {,opt }}^{+} \approx 11$ holds, of providing a single figure of merit, $m_{\ell}$, to quantify the maximum performance of a given riblet, as evidenced in figure 13.

We should note that the collapse observed in figure 13 is purely empirical, and for conventional riblet geometries with clearly defined, open grooves fully exposed to 
the outer flow. Beyond these conventional configurations, $\ell_{g}^{+}$may not necessarily be an adequate parameter to characterize performance, for instance for the fibers and seal fur mentioned above, for which a groove cross-section is difficult to define, or the Tshaped riblets cited by Walsh (1990b). Taking the latter as example, it is clear that, as the wall-parallel segments of the T-fences widen and close into each other, the grooves become increasingly isolated from the overlying flow, while maintaining $\ell_{g}^{+}$essentially constant. In the limit of fully sealed grooves, the geometry would behave as a flat surface, and modifying $\ell_{g}^{+}$would have no performance impact. The merit of $m_{\ell}$ as a single predicting parameter can therefore only be considered an approximation, valid for geometries that are not too different from the experimental 'conventional' riblets -the triangular, trapezoidal, blade, or scalloped riblets frequently proposed in the literature.

\subsection{The breakdown of the viscous regime}

As the riblet size increases, the predictions of the viscous theory break down, particularly the linear dependence of the drag on the size. Goldstein and Tuan (1998) suggested that the deterioration was due to the generation of secondary streamwise vorticity over the riblets, as the unsteady crossflow separates and sheds small-scale vortices that create extra dissipation. However, it is known that spanwise oscillations of the wall, which also presumably introduce unsteady streamwise vorticity, can decrease drag (Jung et al.; 1992), and that modifying the spanwise boundary condition to inhibit the creation of secondary wall vorticity increases drag (Jiménez and Pinelli; 1999). Both observations suggest that introducing small-scale streamwise vorticity near the wall decreases drag by damping the larger streamwise vortices of the buffer layer, and that inertial crossflow effects need not be detrimental to drag reduction. García-Mayoral (2011) considered if the concept of protrusion height could be extended beyond the strictly viscous regime, and that the observed deviations from linearity would be due to the increased importance of advection in the homogeneous-shear-driven models. The results did however not show a significant degradation in the range observed in experiments. Other authors considered that the observed optimum spacing was related to the scale of the turbulent structures in the unperturbed turbulent wall region. Choi et al. (1993), Suzuki and Kasagi (1994) and Lee and Lee (2001), observed that the streamwise turbulent vortices lodge within the riblet grooves for riblets in the drag-degraded regime, and conjectured that this lodging could be responsible for the degradation. García-Mayoral and Jiménez (2011b), studied this effect and observed that, although the vortex lodging eventually occurred, it did so for riblet sizes beyond the onset of degradation.

The above hypotheses were based on observations at spacings for which the viscous regime has already broken down, rather than at those close to the deterioration. As a consequence, it was difficult to establish whether the observed phenomena were consequences or causes of the breakdown. Moreover, although they suggested plausible reasons for why the Stokes regime fails beyond a certain riblet size, none of them provided convincing physical arguments for why that failure should lead to a drag 


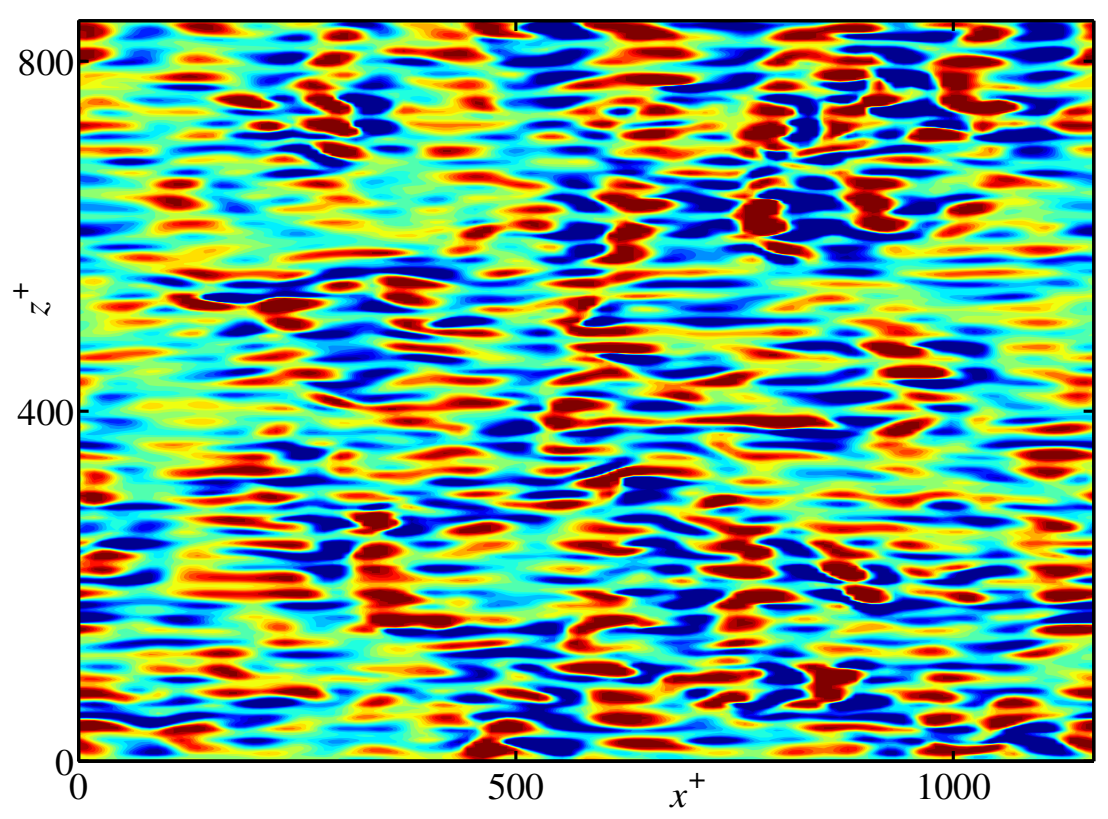

Figure 14. Instantaneous realization of the wall-normal fluctuating velocity at a plane $y^{+} \approx 3$, in a channel with riblets with $\ell_{g}^{+} \approx 17$, larger than the optimum, at $\operatorname{Re}_{\tau} \approx 180$, from García-Mayoral and Jiménez (2011b). Blue regions portray negative velocities, or flow towards the wall, and red regions positive velocities, in the range $-0.5 u_{\tau}-0.5 u_{\tau}$.

increase.

García-Mayoral and Jiménez (2011b) proposed that the degradation in riblet performance with increasing size was caused by the appearance of spanwise-elongated, roll-like structures in the immediate vicinity of the wall, $y^{+} \lesssim 25$, originating from a Kelvin-Helmholtz-like instability of the mean flow. In their DNSs, the instability was observed to trigger only for riblets larger than the optimum, for which the spanwise structures had a distinct footprint in the energy spectrum of the different flow variables, allowing for their quantitative, statistical analysis. Figure 14 portrays an instantaneous realization of the wall-normal velocity at a $y$-plane slightly above the peaks of riblets with $\ell_{g}^{+} \approx 17$, for which the spanwise rollers are fully developed. Just above the riblet peaks the wall-normal velocity is essentially zero, generating streamwise-aligned stripes of very low $v$ separated from each other by the riblet grooves. Above the grooves, where $v$ can attain higher values, the rollers can be observed in the form of spanwise-coherent regions of alternated up- and down-wash flow. These regions can span a substantial number of grooves, in spite of being disrupted with riblet-spacing periodicity by the low- $v$ regions above the peaks.

For riblets with $\ell_{g}^{+}>\ell_{g, \text { opt }}^{+}$, the spanwise rollers have a distinct signature on the spectra in the region with $\lambda_{x}^{+} \approx 100-200$ and $\lambda_{z}^{+}>100-1500$, reaching up to $y^{+} \approx 20-25$ (García-Mayoral and Jiménez; 2011b; García-Mayoral and Jiménez; 2012). This distinct signature could be used to isolate their contribution to drag. A breakdown of different contributions to $\Delta U^{+}$can be derived from the mean momentum equation of a riblet and a reference smooth-wall case. Besides a correction term owing 


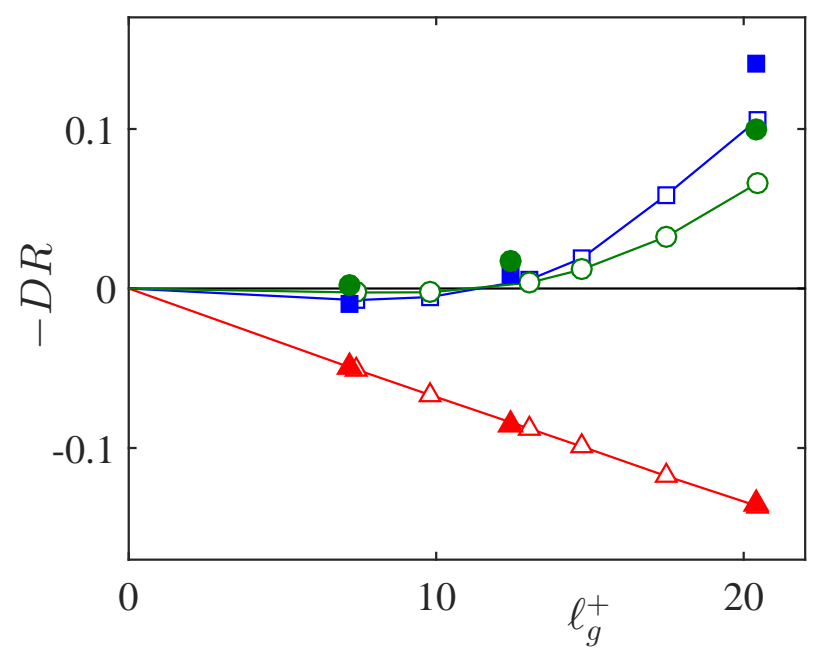

Figure 15. Different contributions to the change in drag for a rectangular riblet from García-Mayoral and Jiménez (2012). $\quad \Delta$, contribution from the slip-length mechanism; $\square$, contribution from additional Reynolds stresses across the whole channel; $\mathrm{O}$, contribution from additional Reynolds stresses restricted to the spectral region occupied by the footprint of spanwise rollers. Open symbols are from DNSs at $\operatorname{Re}_{\tau} \approx 180$, and full symbols at $\operatorname{Re}_{\tau} \approx 550$.

to small differences in height and mass flux of the channels, two essential contributions could be identified. The first is the drag-decreasing contribution from the slip effect discussed in section 3. The second, drag-increasing one, accounted for the additional Reynolds stresses across the whole channel, compared to those over a smooth wall. The results, portrayed in figure 15 show that the latter is the term responsible for the drag degradation for riblets larger than $\ell_{g}^{+} \approx 11$, increasing rapidly for larger sizes and completely canceling the drag-reducing effect of the slip contribution for $\ell_{g}^{+} \approx 20$. Figure 15 also shows the result of restricting the Reynolds-stress contribution to the spectral region associated with the spanwise rollers. It shows that this region contains most of the extra stress, roughly $65 \%-75 \%$, supporting the notion that the rollers are indeed the root cause of the degradation of the drag.

The spanwise rollers reported in García-Mayoral and Jiménez (2011b) and GarcíaMayoral and Jiménez (2012) are similar to those reported over plant canopies (Raupach et al.; 1996; Finnigan; 2000), and over permeable (Jiménez et al.; 2001) and porous walls (Breugem et al.; 2006). They are a prevalent feature of obstructed shear flows (Ghisalberti; 2009), develop easily if the impermeability condition of smooth walls is relaxed, and have been typically attributed to Kelvin-Helmholtz-like instabilities of the mean streamwise flow. Based on this, García-Mayoral and Jiménez (2011b) proposed a simplified stability model to capture the essential physics involved, including the effect of the riblet geometry on the instability. Since the spanwise rollers are quasi-twodimensional in the streamwise and wall-normal directions, the model was spanwisehomogeneous. Viscosity was also neglected, given that Kelvin-Helmholtz instabilities are essentially inviscid, and viscosity would only have a damping, modulating effect. The 
presence of the riblets was modeled in a spanwise-averaged sense through an impedance boundary condition, constructed from the quasi-creeping nature of the flow within the grooves. The resulting model introduced a parameter in the form of a penetration length or 'hydraulic radius,' $\ell_{H}$, measuring how easily the flow can advance through the grooves. When the model was applied on turbulent base flows, the unstable modes obtained agreed reasonably well with the observed wavelengths and shapes of the perturbation. The onset of the instability was shown to depend critically on the value of $\ell_{H}$, measured in wall units. For conventional riblets, $\ell_{H}$ was shown to correlate well with empirical parameter $\ell_{g}^{+}$, with the instability setting in for a value corresponding roughly to $\ell_{g \text {,opt }}^{+}$. This suggests that the empirically fitted $\ell_{g}$, used in $m_{\ell}$ to estimate the performance of riblets a priori, could be substituted by $\ell_{H}$, which is based on the analysis of the physical mechanisms, and should therefore remain valid for unconventional riblets for which predictions based on $\ell_{g}$ are bound to fail.

The identification of the breakdown of the viscous regime as an effect of an instability of the outer flow, triggered by its ability to penetrate the riblet grooves, and with well defined streamwise wavelengths, opens some promising paths for the design of novel configurations, with delayed breakdowns and higher $D R_{\max }$. For instance, geometries that are relatively permeable to the shear flow that defines the protrusion height, but offer more resistance to pressure-driven ones, should be investigated. Another promising area of investigation would be streamwise varying riblets, for instance sinusoidal ones. Such geometries may disrupt the onset of the instability and delay the formation of rollers, and with it the breakdown of the viscous regime. Typically, sinusoidal riblets have not been able to yield better performance than their streamwiseuniform counterparts (Kramer et al.; 2010). One likely reason is that they have generally been designed with very large streamwise wavelengths, aiming to emulate the drag-reducing properties of oscillating walls (Viotti et al.; 2009). Unfortunately, the oscillation induced by sinusoidal riblets on the flow is too weak to produce an oscillatingwall effect. However, sinusoidal riblets could also be designed with shorter wavelengths, comparable to those of spanwise rollers, aiming to inhibit their formation instead. The results of Sasamori et al. (2014) for riblets with streamwise wavelengths of $\sim 360$ wall units would suggest that this is indeed the case. They observed a delay in the mean optimum spacing from $s^{+} \approx 18$ to $s^{+} \approx 30$, resulting in a maximum drag reduction improvement from $\sim 10 \%$ to $\sim 12 \%$.

\section{Anisotropically permeable surfaces}

The understanding gained on the dynamical mechanisms at play for riblets, i.e. the drag reduction induced by the slip effect and its eventual saturation due to the formation of Kelvin-Helmholtz rollers, has motivated research on novel surfaces that maximize the beneficial effect and minimize the deleterious one. In this framework, recent research has been conducted on anisotropically permeable surfaces. The idea is a substrate with high permeability in the streamwise direction, to maximize the streamwise slip effect, 
low permeability in the spanwise direction, to minimize the spanwise slip effect, and low permeability in the wall-normal direction, to minimize the transpiration that gives rise to the Kelvin-Helmholtz rollers. The latter are pervasive in flows over permeable substrates (Jiménez et al.; 2001; Breugem et al.; 2006; Kuwata and Suga; 2016; Zampogna and Bottaro; 2016; Suga et al.; 2017). The large increase in Reynolds stress and subsequent increase in drag that these substrates experience is generally associated to the presence of rollers, which highlights the need to delay their onset as much as possible.

Some previous work existed in the area of permeable substrates to reduce turbulent drag. Hahn et al. (2002) conducted DNSs of turbulent flows over porous substrates which were permeable in the streamwise and/or spanwise directions only. They observed that the streamwise slip was beneficial for drag reduction, while the spanwise slip was adverse. Their substrates, however, were idealized as impermeable in the wall-normal direction, not permitting any transpiration. In that sense, their substrates are probably closer to the slip-only simulations of Min and Kim (2004) and Busse and Sandham (2012) than to realistic permeable coatings, where any measurable streamwise slip at the surface would require momentum transfer with the substrate, and therefore some degree of transpiration.

In Abderrahaman-Elena and Garcia-Mayoral (2017), the slip lengths caused by a permeable substrate were resolved analytically. The flow within the substrate was modeled using a continuum approach, which would formally rely in homogenization techniques (Zampogna and Bottaro; 2016; Lācis and Bagheri; 2017). Two limits were considered, poorly connected substrates and highly connected substrates. In the first case, the flow within neighboring 'pores' would be completely separate, so there would be no diffusive effects over scales larger than the pore size. In the second case, the interconnectivity between pores would be high, and macroscale diffusion over scales larger than the pores would be as efficient as in the free flow. The former can be pictured as a network of microducts, and the latter as a matrix of vanishingly small obstacles, for instance. The flow was modeled using the classical Darcy equations, adding a Brinkman term to account for macroscale diffusivity,

$$
-\nabla p-\nu \mathbf{K}^{-1} \mathbf{u}+\tilde{\nu} \nabla^{2} \mathbf{u}=0
$$

where $\tilde{\nu}$ is the effective macroscale viscosity, which would be $\tilde{\nu} \approx \nu$ for highly connected substrates and $\tilde{\nu} \approx 0$ for poorly connected ones (Taylor; 1971; Auriault; 2009). The permeability tensor $\mathbf{K}$ has components $K_{x}, K_{y}$ and $K_{z}$, which we assume to be along its principal directions. Abderrahaman-Elena and Garcia-Mayoral (2017) argued that highly connected substrates would be more efficient at producing slip lengths, and derived expressions for their slip lengths from (13). For sufficiently large substrate depths, $h^{+} \gtrsim \sqrt{K_{x}^{+}}$, they are

$$
\ell_{x}^{+} \approx \sqrt{K_{x}^{+}}, \quad \quad \ell_{z}^{+} \approx \sqrt{K_{z}^{+}}
$$

The above result indicates that, to achieve drag reduction, substrates with streamwise preferential permeability would be desirable. Drag reduction would still be possible for $K_{x}^{+}=K_{z}^{+}$, as has been observed for some configurations in Rosti et al. (2018), but it 
would rely on the saturation of the effect of $\ell_{z}^{+}$discussed in section 3 , so it requires an unrealistically low wall-normal permeability $K_{z}^{+}$to achieve vanishing transpiration. Such coatings are again more similar to the idealised substrate of Hahn et al. (2002) or the slip-only models of Min and Kim (2004) and Busse and Sandham (2012).

Abderrahaman-Elena and Garcia-Mayoral (2017) also conducted a linear stability analysis to predict the onset of the Kelvin-Helmholtz instability, based on a Darcy model for the substrate, and found that the instability depended essentially on $\sqrt{K_{x}^{+} K_{y}^{+}}$, and would set in for $\sqrt{K_{x}^{+}}=1-5$. Gómez-de-Segura et al. (2018b) extended the analysis of the instability to include macroscale diffusive effects within the substrate and viscous effects within the free flow. They found that for substrates with streamwise preferential permeability, the instability was essentially governed by the wall-normal permeability alone, while when the permeability was preferential in the wall-normal direction the scaling with $\sqrt{K_{x}^{+} K_{y}^{+}}$of Abderrahaman-Elena and Garcia-Mayoral (2017) was recovered. Results for the amplification of the Kelvin-Helmholtz instability from Gómez-de-Segura et al. (2018b) are portrayed in figure 16(a) showing a good collapse as a function of the transpiration length $\ell_{p}^{+} \approx \sqrt{K_{y}^{+}}$for different substrate configurations. Gómez-de-Segura et al. (2018b) also reported results from preliminary DNSs, where the presence of the permeable substrate was modeled through boundary conditions for the overlying flow provided by the analytical solution of (13) within. The simulations confirmed the drag reducing capability of anisotropic substrates.

From the theoretical predictions for the slip lengths of (14) and the results of the stability analysis, Gómez-de-Segura et al. (2018b) produced a map of predicted $\Delta U^{+}$ for substrates with $K_{x}>K_{y}=K_{z}$, with the underlying premise that given a realizable anisotropy ratio, $\phi_{x y}^{2}=K_{x} / K_{y}$, for a given $K_{x}$ it would be desirable to minimize both $K_{y}$ and $K_{z}$ as much as possible, i.e. to the same low value. The predicted values for $\Delta U^{+}$would be limited by the onset of the Kelvin-Helmholtz instability, when a critical value of $\ell_{p}^{+} \approx\left(K_{y}^{+}\right)^{1 / 2}$ is reached. Based on the evolution of the amplification with $\ell_{p}^{+}$, shown in figure 16(a), and from comparison with the analogous analysis for riblets (García-Mayoral and Jiménez; 2011b), a tentative limit was set at $\left(K_{y, \lim }^{+}\right)^{1 / 2}=1-5$.

Based on these predictions, Gómez-de-Segura and Garcia-Mayoral (2019) have conducted a campaign of DNSs focusing on three coatings with different anisotropy ratios, which expressed as ratios of lengthscales were $\phi_{x y} \approx 3.5,5.5$, and 11.5. These were judged to be realistic from a materials and manufacturing perspective. For each configuration, several DNSs were run to measure $\Delta U^{+}$as a function of a Reynolds number based on the texture size, for instance $\left(K_{x}^{+}\right)^{1 / 2}$ or $\left(K_{y}^{+}\right)^{1 / 2}$. From these results, drag curves were produced, similar to those for riblets in figures 12 and 13 . The curves are shown in figure 17. The results show that $\Delta U^{+}$is consistent with the theoretical prediction, $\Delta U^{+}=\ell_{x}^{+}-\ell_{z}^{+} \approx \sqrt{K_{x}^{+}}-\sqrt{K_{z}^{+}}$, until $\Delta U^{+}$peaks roughly at $\left(K_{y, \text { opt }}^{+}\right)^{1 / 2} \approx 0.3$, as it would in riblets for $\ell_{g \text {,opt }}^{+} \approx 11$. No saturation of the effect of the spanwise slip was observed, in contrast with slip-only surfaces. This is consistent with the non-zero transpiration permitted by the wall-normal permeability, which would set a virtual origin $\ell_{v}^{+} \approx\left(K_{y}^{+}\right)^{1 / 2}$. As $\left(K_{y}^{+}\right)^{1 / 2}$ increases beyond $\left(K_{y, \text { opt }}^{+}\right)^{1 / 2}$, eventually the drag 

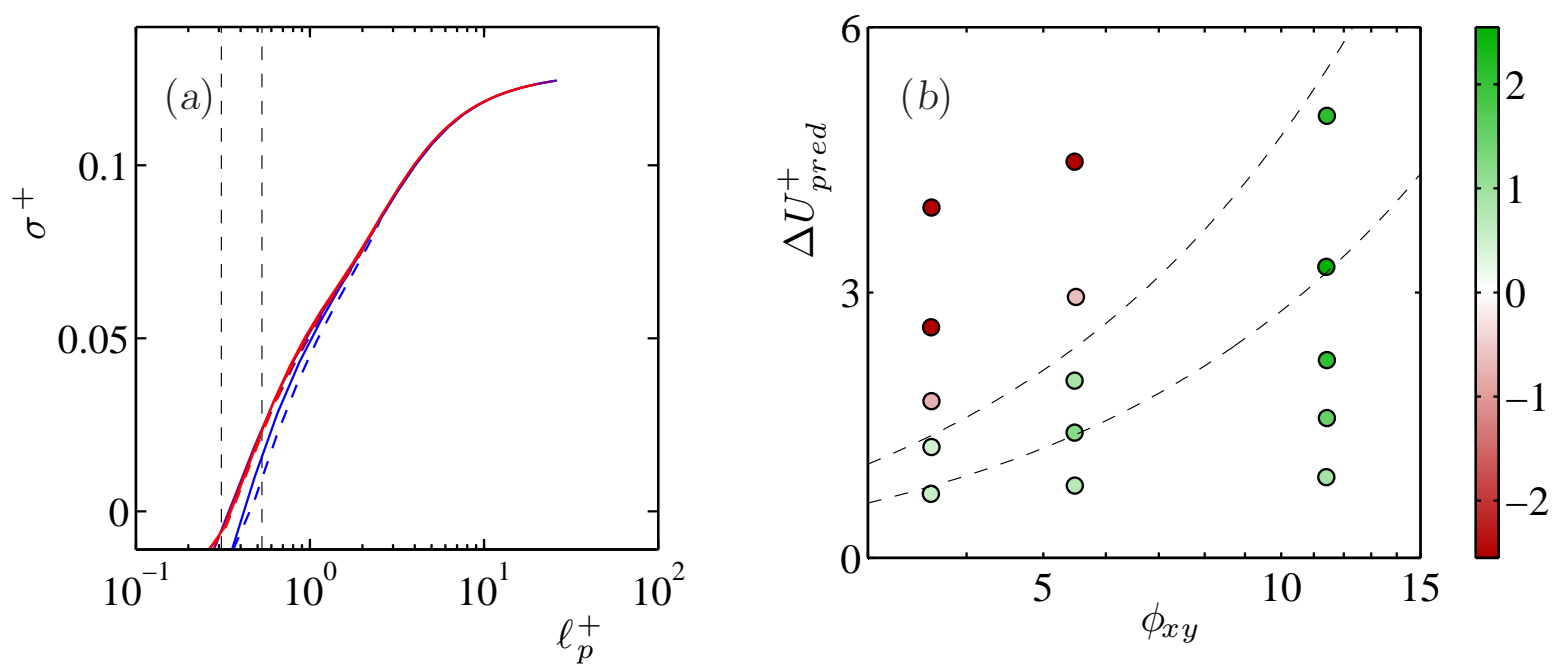

Figure 16. (a) Amplification of Kelvin-Helmholtz-like modes from the linear stability analysis of the flow over permeable substrates with different anisotropy ratios and depths, as a function of $\ell_{p}^{+} \approx \sqrt{K_{y}^{+}}$. (b) Drag predictions, $\Delta U_{p r e d}^{+}$for different anisotropic permeable coatings, as a function of the anisotropy ratio $\phi_{x y}=\sqrt{K_{x} / K_{y}}$. based on their slip lengths; $O$, configurations studied with DNS (Gómez-de-Segura and Garcia-Mayoral; 2019), with the color of the symbols corresponding to the actual $\Delta U^{+}$ measured in the simulations. The dashed lines correspond to $\ell_{p}^{+} \approx 0.30$ and 0.55 .

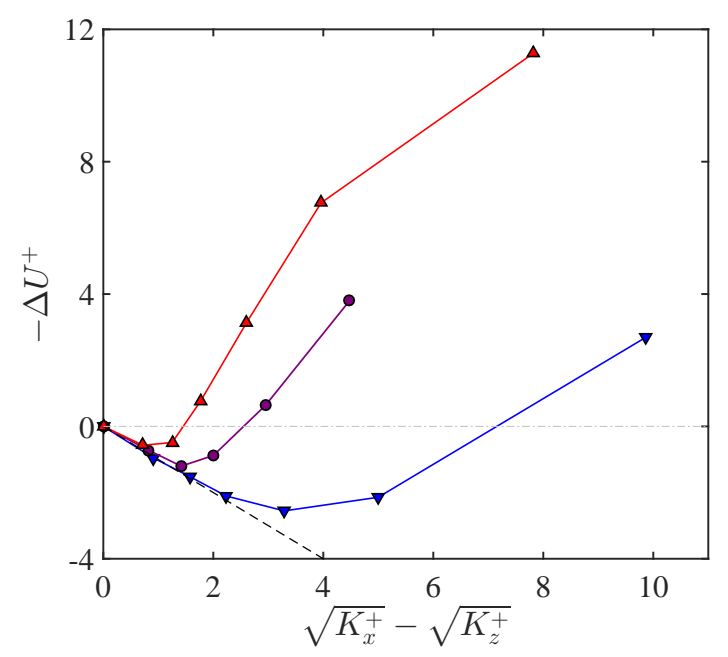

Figure 17. Drag reduction curves for isotropic permeable surfaces from DNS (Gómezde-Segura and Garcia-Mayoral; 2019). $\triangle, \phi_{x y} \approx 3.5 ; \bigcirc, \phi_{x y} \approx 5.5 ; \nabla, \phi_{x y} \approx 11.5$. The dashed line corresponds to the theoretical prediction $\Delta U^{+}=\ell_{x}^{+}-\ell_{z}^{+} \approx \sqrt{K_{x}^{+}}-$ $\sqrt{K_{z}^{+}}$. 

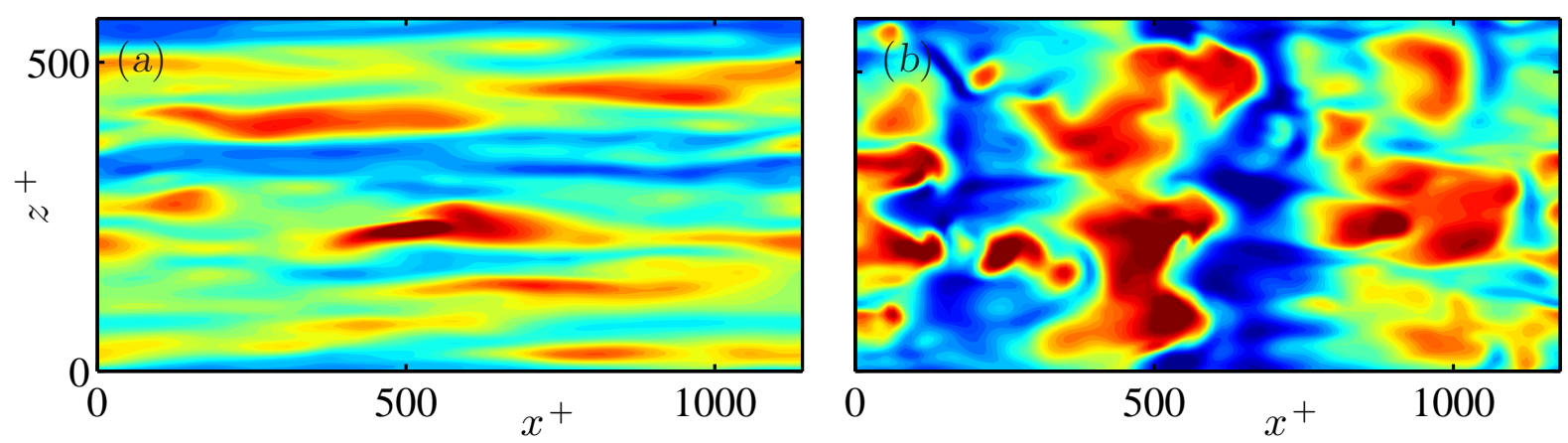

Figure 18. Instantaneous realization of the streamwise fluctuating velocity at a plane $y^{+} \approx 3$, in DNS channels with anisotropic permeable substrates with $\phi_{x y} \approx 11.5$, at $\operatorname{Re}_{\tau} \approx 180$ Gómez-de-Segura and Garcia-Mayoral (2019). (a) $\sqrt{K_{x}^{+}} \approx 1.7$ and $\sqrt{K_{y}^{+}} \approx 0.16 ;(b) \sqrt{K_{x}^{+}} \approx 11$ and $\sqrt{K_{y}^{+}} \approx 0.95$. Blue regions portray negative fluctuations, and red regions positive fluctuations, in the range $-4 u_{\tau}-4 u_{\tau}$.

reduction vanishes, and the smooth-wall drag is recovered for roughly $\left(K_{y, \mathrm{lim}}^{+}\right)^{1 / 2} \approx 0.55$. The results from the DNSs are consistent with the appearance of spanwise-coherent rollers for $K_{y}^{+} \gtrsim K_{y, \text { opt }}^{+}$. For illustration, instantaneous realizations of the streamwise velocity very close to the surface are portrayed in figure 18

Gómez-de-Segura and Garcia-Mayoral (2019) use the DNS results to assess the theoretical predictions of Gómez-de-Segura et al. (2018b). The value $\left(K_{y, \lim }^{+}\right)^{1 / 2} \approx 0.55$ is comparable to, but lower than, the a priori estimate $\left(K_{y, \text { lim }}^{+}\right)^{1 / 2}=1-5$. We can therefore correct the values of $\left(K_{y, \mathrm{lim}}^{+}\right)^{1 / 2}$ and $\left(K_{y, \mathrm{opt}}^{+}\right)^{1 / 2}$ in the prediction map, which is portrayed in figure $16(b)$. Also shown for reference is $\Delta U^{+}$from the DNSs, which shows good agreement for $\left(K_{y}^{+}\right)^{1 / 2} \lesssim\left(K_{y, \text { opt }}^{+}\right)^{1 / 2} \approx 0.3$. It is also worth of notice that the performance of the substrate with $\phi_{x y} \approx 11.5$ reaches $\Delta U^{+} \approx 2.6$, which corresponds to $D R \approx 25 \%$ at the low Reynolds number of the simulations, $\operatorname{Re}_{\tau} \approx 180$. This $D R$ value is at least twice that for the optimal riblet geometries of Bechert et al. (1997a).

The results discussed in this section, especially those of Gómez-de-Segura and Garcia-Mayoral (2019), show great promise and merit further, more detailed investigations. Direct simulations that resolve fully the microstructure of the substrate would be required to test the limits of the models used, and to explore the maximum achievable anisotropy ratios, $\phi_{x y}$ and $\phi_{x y}$, and effective diffusivity, $\tilde{\nu} / \nu$. If the existing results are further confirmed, novel technologies could be tested in the laboratory, taking as a guideline for their design and manufacturing the understanding gained from theory and DNSs. The idea of a substrate highly permeable in the streamwise direction, while impeding more intensely the spanwise and wall normal flow, suggests that a fibrous coating with the fibers aligned in the streamwise direction, as originally proposed by Javier Jiménez for the European FP6 AVERT project (García-Mayoral; 2011), would probably be a suitable technology. 


\section{Conclusions}

In this paper we have reviewed the effect on the overlying flow of textured surfaces that reduce turbulent drag. This effect, when measured directly as a change in drag, depends on the Reynolds number, but is universal when expressed as a shift in the velocity profile away from the surface, expressed in wall units. This shift is therefore the figure to extract from simulations and experiments at low Reynolds number, if the intended application is at a different Reynolds number.

For sufficiently small texture size, we have discussed how the effect of the surface can be reduced to imposing different apparent, virtual origins on the mean velocity profile and on the turbulent eddies. Modifications to the dynamics of near-wall turbulence, often discussed in the literature, can be ascribed entirely to the change in apparent origin, which entails not only a shift in wall normal coordinate but also a change in the friction velocity experienced by the flow, which should be measured at the virtual origin.

As the texture becomes larger, $L^{+} \approx 25$, additional effects appear in addition to the homogenized effect of the virtual origins. The surface induces a texture-coherent flow, modulated in amplitude by the overlying, background shear. For smaller $L^{+}$, this effect is small and does not affect the background turbulence, which remains smooth-like, but for sizes $L^{+} \gtrsim 25$ the background turbulence is modified through its nonlinear coupling with the texture-coherent flow.

In addition to this, textured surfaces often produce dynamical effects beyond the mere modulation of turbulence, which vary greatly from one type of surface to another. We have reviewed the examples of superhydrophobic surfaces and riblets. Superhydrophobic surfaces trigger capillary waves in the air/water interface that propagate upstream and can have a critical impact on the stability of the entrapped air pockets. Riblets excite a Kelvin-Helmholtz-like instability that manifests through the development of spanwise-coherent rollers. These increase mixing and degrade drag. They are a prevalent feature of obstructed shear flows, and develop easily if the notranspiration, impermeability condition of smooth walls is relaxed.

Finally, we have discussed streamwise-preferential permeable surfaces. These have been proposed recently as an improvement over riblets. They generate the same beneficial effect of an offset between origins for the mean flow and the turbulence, and are also susceptible to the formation of Kelvin-Helmholtz rollers, but are more robust than riblets and preliminary evidence suggests that they can provide at least twice as much drag reduction. Further research is however required on this technology to confirm and extend the understanding gained so far.

\section{Acknowledgements}

This work was supported by the International Project Research, 'Fluid Dynamics of Near-Wall Turbulence', promoted in 2016 by the Research Institute for Mathematical 
Sciences (RIMS), a Joint Usage/Research Center located in Kyoto University. Garazi Gómez-de-Segura was supported by Fundación Bancaria 'La Caixa,' and Chris Fairhall was supported by the Engineering and Physical Sciences Research Council, UK.

The authors would like to thank Angela Busse and Neil Sandham for kindly sharing the data of Busse and Sandham (2012).

\section{References}

Abderrahaman-Elena, N. and Garcia-Mayoral, R. (2016). Geometry-induced fluctuations in the transitionally rough regime., J. Phys. Conf. Ser. 708: 012009.

Abderrahaman-Elena, N. and Garcia-Mayoral, R. (2017). Analysis of anisotropically permeable surfaces for turbulent drag reduction., Phys. Rev. Fluids 2: 114609.

Aghdam, S. K. and Ricco, P. (2016). Laminar and turbulent flows over hydrophobic surfaces with shear-dependent slip length, Physics of Fluids 28(3): 035109.

Aljallis, E., Sarshar, M. A., Datla, R., Sikka, V. and Jone, A. (2013). Experimental study of skin friction drag reduction on superhydrophobic flat plates in high Reynolds number boundary layer flow, Phys. Fluids 25: 025103.

Auriault, J. L. (2009). On the domain of validity of Brinkman's equation, Transp. Porous Media 79(2): 215-223.

Bechert, D. W. and Bartenwerfer, M. (1989). The viscous flow on surfaces with longitudinal ribs, J. Fluid Mech. 206: 105-129.

Bechert, D. W., Bruse, M., Hage, W., der Hoeven, J. G. T. V., and Hoppe, G. (1997a). Experiments on drag-reducing surfaces and their optimization with adjustable geometry, J. Fluid Mech. 338: 59-87.

Bechert, D. W., Bruse, M., Hage, W. and Meyer, R. (1997b). Biological surfaces and their technological application - Laboratory and flight experiments on drag reduction and separation control, AIAA Pap. 97-1960.

Boomsma, A. and Sotiropoulos, F. (2016). Direct numerical simulation of sharkskin denticles in turbulent channel flow, Physics of Fluids 28(3): 035106.

Breugem, W. P., Boersma, B. J. and Uittenbogaard, R. E. (2006). The influence of wall permeability on turbulent channel flow, J. Fluid Mech. 562: 35-72.

Bruse, M., Bechert, D. W., der Hoeven, J. G. T. V., Hage, W. and Hoppe, G. (1993). Experiments with conventional and with novel adjustable drag-reducing surfaces, in R. M. C. So, C. G. Speziale and B. E. Launder (eds), Near-Wall Turbulent Flows, Elsevier, pp. 719-738.

Bushnell, D. M. (2003). Aircraft drag reduction - A review, Proc. Instn Mech. Engrs $217(\mathrm{G} 1): 1-18$.

Busse, A. and Sandham, N. D. (2012). Influence of an anisotropic slip-length boundary condition on turbulent channel flow., Phys. Fluids 24: 055111. 
Busse, A. and Sandham, N. D. (2013). Turbulent flow over superhydrophobic surfaces - roughness versus slip, 14TH European Turbulence Conference, Lyon, France.

Busse, A., Sandham, N. D., McHale, G. and Newton, M. I. (2013). Change in drag, apparent slip and optimum air layer thickness for laminar flow over an idealised superhydrophobic surface, Journal of Fluid Mechanics 727: 488-508.

Choi, H., Moin, P. and Kim, J. (1993). Direct numerical simulation of turbulent flow over riblets, J. Fluid Mech. 255: 503-539.

Choi, K.-S. (1989). Near-wall structure of a turbulent boundary layer with riblets, J. Fluid Mech. 208: 417-458.

Choi, K.-S. (2000). European drag-reduction research - Recent developments and current status, Fluid Dynamics Research 26: 325-335.

Chu, D. C. and Karniadakis, G. E. M. (1993). A direct numerical simulation of laminar and turbulent flow over riblet-mounted surfaces, J. Fluid Mech. 250: 1-42.

Clauser, F. (1956). The turbulent boundary layer, Advances Appl. Mech. 4: 1-51.

Daniello, R., Waterhouse, N. E. and Rothstein, J. P. (2009). Turbulent drag reduction using superhydrophobic surfaces, Phys. Fluids 21: 085103.

El-Samni, O. A., Chun, H. H. and Yoon, H. S. (2007). Drag reduction of turbulent flow over thin rectangular riblets, Int. J. Eng. Science 45: 436-454.

Fairhall, C. T. and García-Mayoral, R. (2017). The application of slip length models to larger textures in turbulent flows over superhydrophobic surfaces, Proc. 70th Annual Meeting of the APS Division of Fluid Dynamics.

Fairhall, C. T. and García-Mayoral, R. (2018). Spectral analysis of slip-length model for turbulence over textured superhydrophobic surfaces, Flow, Turbulence and Combustion 100: 961-978.

Finnigan, J. (2000). Turbulence in plant canopies, Ann. Rev. Fluid Mech. 32: 519-571.

Frohnapfel, B., Hasegawa, Y. and Quadrio, M. (2012). Money versus time: evaluation of flow control in terms of energy consumption and convenience, Journal of Fluid Mechanics 700: 406-418.

Fukagata, K., Kasagi, N. and Koumoutsakos, P. (2006). A theoretical prediction of friction drag reduction in turbulent flow by superhydrophobic surfaces., Phys. Fluids 18: 051703 .

García-Mayoral, R. (2011). The interaction of riblets with wall-bounded turbulence, $\mathrm{PhD}$ thesis, School of Aeronatics, UPM, Madrid.

García-Mayoral, R. and Jiménez, J. (2011a). Drag reduction by riblets, Phil. Trans. R. Soc. A 369: 1412-1427.

García-Mayoral, R. and Jiménez, J. (2011b). Hydrodynamic stability and breakdown of the viscous regime over riblets, J. Fluid Mech. 678: 317-347.

García-Mayoral, R. and Jiménez, J. (2012). Scaling of turbulent structures in riblet channels up to $\mathrm{re}_{\tau} \approx 550$, Physics of Fluids 24(10): 105101. 
Gatti, D. and Quadrio, M. (2016). Reynolds-number dependence of turbulent skinfriction drag reduction induced by spanwise forcing, Journal of Fluid Mechanics 802: $553-582$.

Ghisalberti, M. (2009). Obstructed shear flows: similarities across systems and scales, Journal of Fluid Mechanics 641: 51-61.

Gogte, S., Vorobieff, P., Truesdell, R., Mammoli, A., Swol, F. V., Shah, P. and Brinker, C. J. (2005). Effective slip on textured superhydrophobic surfaces, Phys. Fluids 17: 051701 .

Goldstein, D. B., Handler, R. and Sirovich, L. (1995). Direct numerical simulation of turbulent flow over a modeled riblet covered surface, J. Fluid Mech. 302: 333-376.

Goldstein, D. B. and Tuan, T. C. (1998). Secondary flow induced by riblets, J. Fluid Mech. 363: 115-151.

Gómez-de-Segura, G., Fairhall, C. T., MacDonald, M., Chung, D. and Garcia-Mayoral, R. (2018a). Manipulation of near-wall turbulence by surface slip and permeability, $J$. Phys. Conf. Ser. 1001: 012011.

Gómez-de-Segura, G., Sharma, A. and Garcia-Mayoral, R. (2018b). Turbulent drag reduction using anisotropic permeable substrates, Flow, Turbulence and Combustion 100: $995-1014$.

Gómez-de-Segura, G. and Garcia-Mayoral, R. (2019). Turbulent drag reduction by anisotropic permeable surfaces - analysis and direct numerical simulations, In preparation .

Gose, J. W., Golovin, K., Boban, M., Mabry, J. M., Tuteja, A., Perlin, M. and Ceccio, S. L. (2018). Characterization of superhydrophobic surfaces for drag reduction in turbulent flow, Journal of Fluid Mechanics 845: 560-580.

Grüneberger, R. and Hage, W. (2011). Drag characteristics of longitudinal and transverse riblets at low dimensionless spacings, Exp. in Fluids 50: 363-373.

Hage, W., Bechert, D. W. and Bruse, M. (2000). Yaw angle effects on optimized riblets, in P. Thiede (ed.), Proc. of the CEAS/DragNet European Drag Reduction Conf., Springer-Verlag, Potsdam, Germany, pp. 278-285.

Hahn, S., Je, J. and Choi, H. (2002). Direct numerical simulation of a turbulent channel flow with permeable walls, J. Fluid Mech. 450: 259-285.

Hoyas, S. and Jiménez, J. (2006). Scaling of the velocity fluctuations in turbulent channels up to $\mathrm{re}_{\tau}=2003$, Physics of Fluids 18(1): 011702.

Hyväluoma, J. and Harting, J. (2008). Slip flow over structured surfaces with entrapped microbubbles, Phys. Rev. Lett. 100: 246001.

Itoh, M., Tamano, S., Iguchi, R., Yokota, K., Akino, N., Hino, R. and Kubo, S. (2006). Turbulent drag reduction by the seal fur surface, Phys. Fluids 18(6): 065102.

Jelly, T. O., Jung, S. Y. and Zaki, T. A. (2014). Turbulence and skin friction modification in channel flow with streamwise-aligned superhydrophobic surface texture., Phys. Fluids 26: 095102. 
Jiménez, J. (1994). On the structure and control of near wall turbulence, Phys. Fluids 6(2): 944-953.

Jiménez, J. (2004). Turbulent flows over rough walls, Ann. Rev. Fluid Mech. 36: 173196.

Jiménez, J. and Pinelli, A. (1999). The autonomous cycle of near wall turbulence, J. Fluid Mech. 389: 335-359.

Jiménez, J., Uhlman, M., Pinelli, A. and G., K. (2001). Turbulent shear flow over active and passive porous surfaces, J. Fluid Mech. 442: 89-117.

Jung, W., Mangiavacchi, N. and Akhavan, R. (1992). Supression of turbulence in wallbounded flows by high-frequency spanwise oscillations, Phys. Fluids A 4: 1605-1607.

Kordy, H. (2015). Process abilities of the riblet-coating process with dual-cure lacquers, CIRP Journal of Manufacturing Science and Technology 11: 1-9.

Kramer, F., Grüneberger, R., Thiele, F., Wassen, E., Hage, W. and Meyer, R. (2010). Wavy riblets for turbulent drag reduction, Proc. of 5th AIAA Flow Control Conf, AIAA, Chicago, Illinois, USA.

Kramer, M. O. (1937). Einrichtung zur Verminderung des Reibungswiderstandes, Patentschrift 669897, Klasse 62b, Gruppe 408. March 17.

Krieger, K. (2004). Do pool sharks really swim faster?, Science 305: 636-637.

Kuwata, Y. and Suga, K. (2016). Lattice Boltzmann direct numerical simulation of interface turbulence over porous and rough walls, Int. J. Heat Fluid Flow 0: 1-13.

Lācis, U. and Bagheri, S. (2017). A framework for computing effective boundary conditions at the interface between free fluid and a porous medium, J. Fluid Mech. 812: 866-889.

Lauga, J. and Stone, H. (2003). Effective slip in pressure-driven Stokes flow, J. Fluid Mech. 489: 55-77.

Lee, C. and Kim, C.-J. (2009). Maximizing the giant liquid slip on superhydrophobic microstructures by nanostructuring their sidewalls, Langmuir 25: 12812-12818.

Lee, C. and Kim, C.-J. (2011a). Influence of surface hierarchy of superhydrophobic surfaces on liquid slip, Langmuir 27: 4243-4248.

Lee, C. and Kim, C.-J. (2011b). Underwater restoration and retention of gases on superhydrophobic surfaces for drag reduction, Phys. Rev. Lett. 106: 014502.

Lee, C. and Kim, C.-J. (2012). Wetting and active dewetting processes of hierarchically constructed superhydrophobic surfaces fully immersed in water, J. MEMS 21: 712720.

Lee, M. and Moser, R. D. (2015). Direct numerical simulation of turbulent channel flow up to, Journal of Fluid Mechanics 774: 395-415.

Lee, S.-J. and Jang, Y.-G. (2005). Control of flow around a NACA 0012 airfoil with a micro-riblet film, J. Fluids Struct. 20: 659-672. 
Lee, S.-J. and Lee, S.-H. (2001). Flow field analysis of a turbulent boundary layer over a riblet surface, Experiments in Fluids 30: 153-166.

Ling, H., Srinivasan, S., Golovin, K., McKinley, G. H., Tuteja, A. and Katz, J. (2016). High-resolution velocity measurement in the inner part of turbulent boundary layers over super-hydrophobic surfaces, Journal of Fluid Mechanics 801: 670-703.

Liu, Y., Wexler, J. S., Schönecker, C. and Stone, H. A. (2016). Effect of viscosity ratio on the shear-driven failure of liquid-infused surfaces, Physical Review Fluids 1(7).

Lozano-Durán, A. and Jiménez, J. (2014). Effect of the computational domain on direct simulations of turbulent channels up to $\mathrm{re}_{\tau}=4200$, Physics of Fluids 26(1): 011702.

Luchini, P. (1995). Asymptotic analysis of laminar boundary-layer flow over finely grooved surfaces, Europ. J. of Mechanics B/Fluids 14: 169-195.

Luchini, P. (1996). Reducing the turbulent skin friction, Computational Methods in Applied Sciences - Proc. 3rd ECCOMAS CFD Conference, pp. 466-470.

Luchini, P. (2015). The relevance of longitudinal and transverse protrusion heights for drag reduction by a superhydrophobic surface, Proceedings of the 2015 European Drag Reduction and Flow Control Meeting.

Luchini, P., Manzo, F. and Pozzi, A. (1991). Resistance of a grooved surface to parallel flow and cross-flow, J. Fluid Mech. 228: 87-109.

MacDonald, M., Chung, D., Hutchins, N., Chan, L., Ooi, A. and García-Mayoral, R. (2017). The minimal-span channel for rough-wall turbulent flows, Journal of Fluid Mechanics 816: 5-42.

MacDonald, M., Ooi, A., García-Mayoral, R., Hutchins, N. and Chung, D. (2018). Direct numerical simulation of high aspect ratio spanwise-aligned bars, Journal of Fluid Mechanics 843: 126-155.

Marinho, D. A., Reis, V. M., Alves, F. B., Vilas-Boas, J. P., Machado, L., Silva, A. J. and Rouboa, A. I. (2009). The hydrodynamic drag during gliding in swimming, $J$. Appl. Biomech. 25: 253-257.

Martell, M. B., Perot, J. B. and Rothstein, J. P. (2009). Direct numerical simulations of turbulent flows over superhydrophobic surfaces, J. Fluid Mech. 620: 31-41.

Martell, M. B., Rothstein, J. P. and Perot, J. B. (2010). An analysis of superhydrophobic turbulent drag reduction mechanisms using direct numerical simulation, Phys. Fluids 22: 065102 .

Mathis, R., Hutchins, N. and Marusic, I. (2009). Large-scale amplitude modulation of the small-scale structures in turbulent boundary layers, Journal of Fluid Mechanics 628: 311 .

Matthews, J. N. A. (2008). Low-drag suit propels swimmers, Physics Today August: 3233.

Min, T. and Kim, J. (2004). Effects of hydrophobic surface on skin-friction drag, Phys. Fluids 16(7): L55-L58. 
Mizuno, Y. and Jiménez, J. (2011). Mean velocity and length-scales in the overlap region of wall-bounded turbulent flows, Physics of Fluids 23(8): 085112.

Moser, R. D., Kim, J. and Mansour, N. N. (1999). Direct numerical simulation of turbulent channel flow up to $\mathrm{re}_{\tau}=590$, Physics of Fluids 11(4): 943-945.

Orlandi, P. and Jiménez, J. (1994). On the generation of turbulent wall friction, Phys. Fluids 6: 634-641.

$\mathrm{Ou}$, J. and Rothstein, J. P. (2005). Direct velocity measurements of the flow past drag-reducing ultrahydrophobic surfaces, Phys. Fluids 17: 13606.

Park, H., Park, H. and Kim, J. (2013b). A numerical study of the effects of superhydrophobic surface on skin-friction drag in turbulent channel flow, Phys. Fluids 25: 110815.

Park, H., Sun, G. and Kim, C.-J. (2014). Superhydrophobic turbulent drag reduction as a function of surface grating parameters, J. Fluid Mech. 747: 722-734.

Park, S.-R. and Wallace, J. M. (1994). Flow alteration and drag reduction by riblets in a turbulent boundary layer, AIAA J. 32(1): 31-38.

Patankar, N. A. (2010). Consolidation of hydrophobic transition criteria by using an approximate energy minimization approach, Langmuir 26: 8941-8945.

Rastegari, A. and Akhavan, R. (2015). On the mechanism of turbulent drag reduction with super-hydrophobic surfaces., J. Fluid Mech. 773: R4.

Raupach, M. R., Finnigan, J. and Brunet, Y. (1996). Coherent eddies and turbulence in vegetation canopies: the mixing-layer analogy, Boundary-Layer Meteorology 78: 351382 .

Reynolds, W. C. and Hussain, A. K. M. F. (1972). The mechanics of an organized wave in turbulent shear flow. Part 3. Theoretical models and comparisons with experiments, J. Fluid Mech. 54: 263-288.

Robert, J. F. (1992). Drag reduction: an industrial challenge, Skin friction drag reduction, number 786 in AGARD Rep., pp. 2.1-2.15.

Roskam, J. (1987). Airplane design. Part VI: Preliminary calculation of aerodynamic, thrust and power characteristics, Roskam Aviation and Engineering Corporation.

Rosti, M. E., Brandt, L. and Pinelli, A. (2018). Turbulent channel flow over an anisotropic porous wall - drag increase and reduction, Journal of Fluid Mechanics 842: 381-394.

Rothstein, J. P. (2010). Slip on superhydrophobic surfaces, Annu. Rev. Fluid Mech. 42: 89-109.

Samaha, M. A., Vahedi, T. H. and el Hak, M. G. (2011). Modeling drag reduction and meniscus stability of superhydrophobic surface of random roughness, Phys. Fluids 23: 012001.

Sasamori, M., Mamori, H., Iwamoto, K. and Murata, A. (2014). Experimental study on drag-reduction effect due to sinusoidal riblets in turbulent channel flow, Experiments in Fluids 55(10). 
Schönecker, C., Baier, T. and Hardt, S. (2014). Influence of the enclosed fluid on the flow over a microstructured surface in the cassie state, Journal of Fluid Mechanics 740: $168-195$.

Seo, J., Garcia-Mayoral, R. and Mani, A. (2015). Pressure fluctuations in turbulent flows over superhydrophobic surfaces., J. Fluid Mech. 783: 448-473.

Seo, J., Garcia-Mayoral, R. and Mani, A. (2018). Turbulent flows over superhydrophobic surfaces: flow-induced capillary waves, and robustness of air-water interfaces., J. Fluid Mech. 835: 45-85.

Seo, J. and Mani, A. (2016). On the scaling of the slip velocity in turbulent flows over superhydrophobic surfaces., Phys. Fluids 28: 025110.

Sha, T., Itoh, M., Tamano, S., Yokota, K. and Akino, N. (2005). Experimental study on drag reduction in turbulent flow on zigzag riblet surface, Fluids Eng. Conf./Ryutai Kogaku Bumon Koenkai Koen Ronbunshu 83: 207.

Spalart, P. R. and McLean, J. D. (2011). Drag reduction: enticing turbulence, and then an industry, Phil. Trans. R. Soc. A 369: 1556-1569.

Squire, H. B. (1953). Investigation of the instability of a moving liquid film, Brit. J. Appl. Phys. 4: 167-169.

Suga, K., Nakagawa, Y. and Kaneda, M. (2017). Spanwise turbulence structure over permeable walls, J. Fluid Mech. 822: 186-201.

Suzuki, Y. and Kasagi, N. (1994). Turbulent drag reduction mechanism above a riblet surface, AIAA J. 32(9): 1781-1790.

Szodruch, J. (1991). Viscous drag reduction on transport aircraft, AIAA Pap. 91-0685.

Taylor, G. I. (1959). The dynamics of thin sheets of fluid. ii. Waves on fluid sheets, Proc. R. Soc. A 253: 296-312.

Taylor, G. I. (1971). A model for boundary condition of a porous material. Part 1, J. Fluid Mech. 49(2): 310-326.

Tennekes, H. and Lumley, J. L. (1972). A first course in turbulence, MIT Press.

Teo, C. J. and Khoo, B. C. (2010). Flow past superhydrophobic surfaces containing longitudinal grooves: effects of interface curvature, Microfluidics and Nanofluidics 9: $499-511$.

Türk, S., Daschiel, G., Stroh, A., Hasegawa, Y. and Frohnapfel, B. (2014). Turbulent flow over superhydrophobic surfaces with streamwise grooves, J. Fluid Mech. 747: $186-217$.

Viotti, C., Quadrio, M. and Luchini, P. (2009). Streamwise oscillation of spanwise velocity at the wall of a channel for turbulent drag reduction, Phys. Fluids 21: 115109.

Viswanath, P. R. (2002). Aircraft viscous drag reduction using riblets, Progress in Aerospace Sciences 38: 571-600.

Vukoslavcevic, P., Wallace, J. M. and Balint, J.-L. (1992). Viscous drag reduction using streamwise aligned riblets, AIAA J. 30: 1119-1122. 
Walsh, M. J. (1990a). Effect of detailed surface geometry on riblet drag reduction performance, J. of Aircraft 27(6): 572-573.

Walsh, M. J. (1990b). Riblets, in D. M. Bushnell and J. N. Hefner (eds), Viscous drag reduction in boundary layers, AIAA, pp. 203-261.

Walsh, M. J. and Lindemann, A. M. (1984). Optimization and application of riblets for turbulent drag reduction, AIAA Pap. 84-0347.

Wenzel, R. N. (1936). Resistance of solid surfaces to wetting by water, Ind. Eng. Chem. 28: $988-994$.

Woolford, B., Prince, J., Maynes, D. and Webb, B. W. (2009). Particle image velocimetry characterization of turbulent channel flow with rib patterned superhydrophobic walls, Physics of Fluids 21(8): 085106.

Ybert, C., Barentin, C. and Cottin-Bizonne, C. (2007). Achieving large slip with superhydrophobic surfaces: Scaling laws for generic geometries, Phys. Fluids 19: 123601.

Zampogna, G. A. and Bottaro, A. (2016). Fluid flow over and through a regular bundle of rigid fibres, J. Fluid Mech. 792: 5-35.

Zhang, C. and Chernyshenko, S. I. (2016). Quasisteady quasihomogeneous description of the scale interactions in near-wall turbulence, Phys. Rev. Fluids 1: 014401. 